\title{
Activity Patterns and Synaptic Organization of Ventrally Located Interneurons in the Embryonic Chick Spinal Cord
}

\author{
Amy Ritter, Peter Wenner, Stephen Ho, Patrick J. Whelan, and Michael J. O'Donovan \\ Section on Developmental Neurobiology, Laboratory of Neural Control, National Institute of Neurological Disorders and \\ Stroke, National Institutes of Health, Bethesda, Maryland 20892
}

To investigate the origin of spontaneous activity in developing spinal networks, we examined the activity patterns and synaptic organization of ventrally located lumbosacral interneurons, including those whose axons project into the ventrolateral funiculus (VLF), in embryonic day 9 (E9)-E12 chick embryos. During spontaneous episodes, rhythmic synaptic potentials were recorded from the VLF and from spinal interneurons that were synchronized, cycle by cycle, with rhythmic ventral root potentials. At the beginning of an episode, ventral root potentials started before the VLF discharge and the firing of individual interneurons. However, pharmacological blockade of recurrent motoneuron collaterals did not prevent or substantially delay interneuron recruitment during spontaneous episodes. The synaptic connections of interneurons were examined by stimulating the VLF and recording the potentials evoked in the ventral roots, in the VLF, or in individual interneurons. Low- intensity stimulation of the VLF evoked a short-latency depolarizing potential in the ventral roots, or in interneurons, that was probably mediated mono- or disynaptically. At higher intensities, long-latency responses were recruited in a highly nonlinear manner, eventually culminating in the activation of an episode. VLF-evoked potentials were reversibly blocked by extracellular $\mathrm{Co}^{2+}$, indicating that they were mediated by chemical synaptic transmission. Collectively, these findings indicate that ventral interneurons are rhythmically active, project to motoneurons, and are likely to be interconnected by recurrent excitatory synaptic connections. This pattern of organization may explain the synchronous activation of spinal neurons and the regenerative activation of spinal networks when provided with a suprathreshold stimulus.

Key words: spinal cord; rhythmic activity; interneurons; development; synchrony; chick
Spontaneous activity is a characteristic feature of developing networks in many parts of the nervous system and is believed to play an essential role in the refinement of neuronal connectivity (for review, see Katz and Shatz, 1996; O’Donovan, 1999). Despite the importance of this activity, its genesis is poorly understood. We have been studying this problem using an isolated preparation of the chick spinal cord that produces spontaneous episodes of rhythmic activity. Imaging studies in this preparation have shown that rhythmically active neurons are synchronized from cycle to cycle and are widely distributed throughout the lumbosacral cord, with a high concentration dorsomedial to the lateral motor column (O'Donovan et al., 1992, 1994). This synchronous pattern of activation is common in the developing nervous system, but little is known about the cellular and synaptic mechanisms responsible for its generation (Wong et al., 1995; Garaschuk et al., 1998; Schwartz et al., 1998).

Our previous work has indicated that interneurons whose axons project into the ventrolateral funiculus (VLF) contribute to the synchronization and excitatory drive of motoneurons during episodes of rhythmic activity (Ho and O'Donovan, 1993; in the rat, see also Magnuson et al., 1995). However, nothing is known about the activity patterns or connectivity of these or any other ventral interneurons. Indeed, to date there has been no electrophysiolog-

Received July 27, 1998; revised Jan. 12, 1999; accepted Feb. 9, 1999.

A.R. was supported by a National Research Service Award from the National Institutes of Health. P.J.W. was supported by a fellowship from the National Sciences and Engineering Research Council of Canada.

Correspondence should be addressed to Dr. Michael J. O'Donovan, Laboratory of Neural Control, Room 3A50, Building 49, National Institutes of Health, Bethesda, MD 20892.

Copyright (C) 1999 Society for Neuroscience $\quad 0270-6474 / 99 / 193457-15 \$ 05.00 / 0$ ical analysis of the organization of interneuronal networks in the developing spinal cord. Calcium-imaging experiments have shown that the earliest optical activity at the onset of a spontaneous episode occurs in the vicinity of the lateral motor column, raising the intriguing possibility that motoneurons might be involved in triggering spontaneous episodes (O'Donovan et al., 1994). However, because of the indirect nature of calcium imaging and the limited temporal resolution of video (30 frames/sec), these studies could not establish definitively that motoneuron activity preceded that of interneurons at episode onset.

The first goal of the present experiments was to establish the activity patterns of ventral interneurons using intracellular recordings and electrotonic recordings from the VLF. In the second part of the study, we investigated the precise timing of motoneuron and interneuron activity during an episode. Finally, we examined the synaptic organization of the interneuronal networks by recording the potentials evoked in motoneurons and interneurons in response to stimulation of the VLF.

Parts of this paper have been published previously (Ho and O’Donovan, 1992; Ritter and O’Donovan, 1993; O'Donovan and Ritter, 1995a,b).

\section{MATERIALS AND METHODS}

Experiments were performed on the isolated spinal cord of embryonic White Leghorn chicken embryos [embryonic day 9 (E9)-E12]. The lumbosacral spinal cord with attached nerves was dissected under cooled $\left(10^{\circ} \mathrm{C}\right)$ oxygenated Tyrode's solution $(139 \mathrm{~mm} \mathrm{NaCl}, 12 \mathrm{~mm}$ glucose, 17 mM $\mathrm{NaHCO}_{3}, 2.9 \mathrm{~mm} \mathrm{KCl}, 1 \mathrm{~mm} \mathrm{MgCl}$, and $3 \mathrm{~mm} \mathrm{CaCl}_{2}$ ) or, in some experiments, using a sucrose-substituted (Aghajanian and Rasmussen, 1989) Tyrode's solution (278 mM sucrose, $12 \mathrm{~mm}$ glucose, $17 \mathrm{~mm}$ $\mathrm{NaHCO}_{3}, 3 \mathrm{~mm} \mathrm{KCl}, 1 \mathrm{~mm} \mathrm{MgCl}_{2}$, and $0 \mathrm{~mm} \mathrm{CaCl}_{2}$ ).

A strip of the VLF was dissected off the cord with a fine tungsten 
needle either caudal to the crural plexus, from lumbosacral segment 4 (LS4) to LS6 (caudal VLF), or rostrally from T5 or T7 and extending to T7 or T9 (rostral VLF).

The preparation was then transferred to a recording chamber containing normal Tyrode's solution. Neural activity was recorded from muscle nerves or ventral roots and from the rostral or caudal portions of the dissected VLF. The neural recordings were made using tight-fitting suction electrodes connected to high-gain DC amplifiers (Grass P16 or World Precision Instruments DAM70), digitized (Neurocorder DR-886; Neuro Data Instruments), and taped for further analysis. The filter was set at $\mathrm{DC}$ to 1 or $3 \mathrm{kHz}$ for recording slow potentials and at $100 \mathrm{~Hz}$ to 1 or $3 \mathrm{kHz}$ to isolate discharge. The recordings were made at room temperature $\left(20-22^{\circ} \mathrm{C}\right)$ or after heating the perfusate to $27-29^{\circ} \mathrm{C}$.

The VLF was stimulated using square current pulses $(2-50 \mu \mathrm{A}$; $50-500 \mu \mathrm{sec})$. In some experiments, a $\mathrm{Co}^{2+}$ gel was applied around the cord to block synaptic transmission over a restricted region ( $\mathrm{Ho}$ and O'Donovan, 1993). The gel was made with $10 \mathrm{mM} \mathrm{CoCl}_{2}$ in $15 \%$ gelatin (dissolved in warm Tyrode's solution). To apply the gel, we first built a high-vacuum grease barrier around the cord leaving a space where the gel could be injected. The $\mathrm{Co}^{2+}$ gel was then injected into the barrier with a glass pipette and allowed to set. During this time the perfusion was stopped. Typically, the discharge of motoneurons within the gel was blocked within $\sim 20 \mathrm{~min}$.

Whole-cell recordings from motoneurons and interneurons. Whole-cell recordings were obtained from ventrally located interneurons in the lumbosacral cord (Sernagor and O'Donovan, 1991). The cord was secured in the recording chamber with a series of nylon threads or insect pins so that the ventral surface faced upward. Electrodes were pulled in two stages from thin-walled glass using a Brown-Flaming puller. The patch electrode contained one of two solutions (concentrations in $\mathrm{mm}$ ): for $\mathrm{A}, 130 \mathrm{KMeSO}_{3}$, 5 EGTA, $10 \mathrm{HEPES}, 1 \mathrm{ATP}, 1 \mathrm{MgCl}_{2}$, and 0.5 $\mathrm{CaCl}_{2}$, or for B, $140 \mathrm{KGluconate,} 10 \mathrm{HEPES}, 1.1 \mathrm{EGTA}, 0.1 \mathrm{CaCl}_{2}, 0.5$ $\mathrm{MgCl}_{2}$, and $1.0 \mathrm{ATP}$, as well as $0.5 \%$ neurobiotin. Tip resistances were $\sim 4-8 \mathrm{M} \Omega$.

Whole-cell recordings were obtained either after penetrating the ventral white matter or directly from cells in the cut transverse face (O'Donovan et al., 1994). The cut was made at either the LS2, LS3, or LS6 level using a vibrating blade (O'Donovan et al., 1994). As the electrode was advanced to the desired location and depth of the cord, a positive pipette pressure was maintained while hyperpolarizing current pulses were applied to monitor the tip resistance.

Gigaohm seals were formed in current-clamp mode after the application of gentle suction or, in some instances, after the release of the positive pressure. More negative pressure was applied to rupture the membrane and achieve the whole-cell configuration. Only cells showing a stable resting membrane potential more negative than $-40 \mathrm{mV}$ for 15 min or more were used for further analysis. Series resistance was not compensated.

Ten interneurons were identified as projecting into the VLF by antidromic stimulation of the VLF. Other cells were identified as interneurons by positioning the recording electrode outside the lateral motor column (cut-face preparation) and by the absence of antidromic spikes after stimulation of the ventral root or muscle nerve. Some interneurons were filled with neurobiotin (see below) and could be distinguished from motoneurons by their location outside the lateral motor column.

Cell labeling. In some experiments an individual interneuron was injected with $0.5 \%$ neurobiotin. Only a single cell was recorded and injected in a single experiment to allow unequivocal matching of the recording with the stained cell. At the end of the experiments, the spinal cord was fixed in 5\% glutaraldehyde and sectioned at $100 \mu \mathrm{m}$ on a vibratome, and the sections were processed for biocytin/neurobiotin.

Temporal relations between rhythmic synaptic potentials in the interneurons and population electrical activity recorded from muscle nerves and the $V L F$. We examined the temporal relationships between the activity of interneurons and motoneurons by generating cross-correlograms between the synaptic potential of individual interneurons and the slowpotential neurogram from the femorotibialis muscle nerve and, when available, the neurogram from the VLF. The neurograms were low-pass filtered to remove spike activity. The records were then digitized using an HP54600A oscilloscope, converted to an ASCII file using HP Benchlink software, and imported into a spreadsheet (Quattro Pro). DC components and trends were removed from the traces, and they were smoothed with a five point-moving average before cross-correlograms were computed by the use of commercially available software (Statistica).

\section{RESULTS}

\section{Activity patterns of interneurons}

Extracellular suction electrode recordings from the ventrolateral funiculus during episodes of rhythmic activity

Our previous work showed that axons traveling in the VLF contribute to the synchronization and excitatory drive of motoneurons during episodes of rhythmic activity (Ho and O'Donovan, 1993). This observation suggests that some of the cells giving rise to VLF axons are likely to be activated rhythmically during episodes of motoneuron bursting. To test this idea, we made extracellular DC recordings from VLF axons during episodes of rhythmic activity. This technique allows population synaptic potentials to be recorded from the ventral roots that closely resemble the intracellularly recorded membrane potential changes in individual motoneurons (O'Donovan, 1989). When applied to the VLF, these recordings provide information about the summed synaptic drive in a population of interneurons and also reveal the timing of interneuronal discharge (see below). Such recordings made from a section of the VLF peeled off of the cord at LS5-LS6 revealed the presence of rhythmic, population synaptic potentials synchronized cycle by cycle with the rhythmic potentials recorded from ventral roots or muscle nerves (Fig. 1).

To ensure that the VLF potentials were not generated by synaptically activated remnants of gray matter (such as motoneurons) within the teased axonal filaments or by paragriseal neurons within the white matter, we blocked synaptic activity within the dissected VLF using a $\mathrm{Co}^{2+}$ gel around the spinal cord (Fig. $1 B$, see inset). Rhythmic, slow potentials could still be recorded from the VLF after local synaptic activity was blocked by the $\mathrm{Co}^{2+}$ gel ( $n=3$ experiments). Typically, the blockade took $\sim 20 \mathrm{~min}$ to act, and recordings were made $30 \mathrm{~min}$ after application of the gel. The efficacy of synaptic blockade was demonstrated by the virtual abolition of the activity of LS7 motoneurons located under the gel (Fig. $1 A, B$, compare $L S 7$ recordings). As illustrated in Figure 1 , the activity of femorotibialis motoneurons, which lie close to the rostral edge of the gel, could also be depressed presumably because of $\mathrm{Co}^{2+}$ leakage from the gel. Collectively, these findings indicate that the population potentials recorded from the VLF are not generated by synaptically activated neurons lying within the dissected portion of the VLF.

Because the recordings from the VLF are electrotonic potentials generated in a population of interneurons, they are an indirect monitor of the activity of individual interneurons. Therefore, to confirm the VLF recordings and to establish the variability in the activity patterns of individual interneurons, we recorded intracellularly from ventrally located interneurons during episodes of rhythmic activity.

\section{Intracellular recordings from ventrally located interneurons}

We recorded from 122 interneurons, of which 10 were antidromically identified by VLF stimulation (see Materials and Methods). Ninety-seven of the cells were recorded from the cut-face preparation, whereas the reminder (25) were recorded through the ventral surface of the cord. For the 97 cells recorded in the cut-face configuration, input resistance measured $750 \pm 380 \mathrm{M} \Omega$. The electrode tip was well below the surface of the perfusate in the cut-face recording, precluding the use of discontinuous current clamp. As a result, these measurements are uncorrected for series resistance. The input impedance of 16 cells that were penetrated through the ventral surface of the cord and were recorded with discontinuous current clamp was $590 \pm 310 \mathrm{M} \Omega$. 

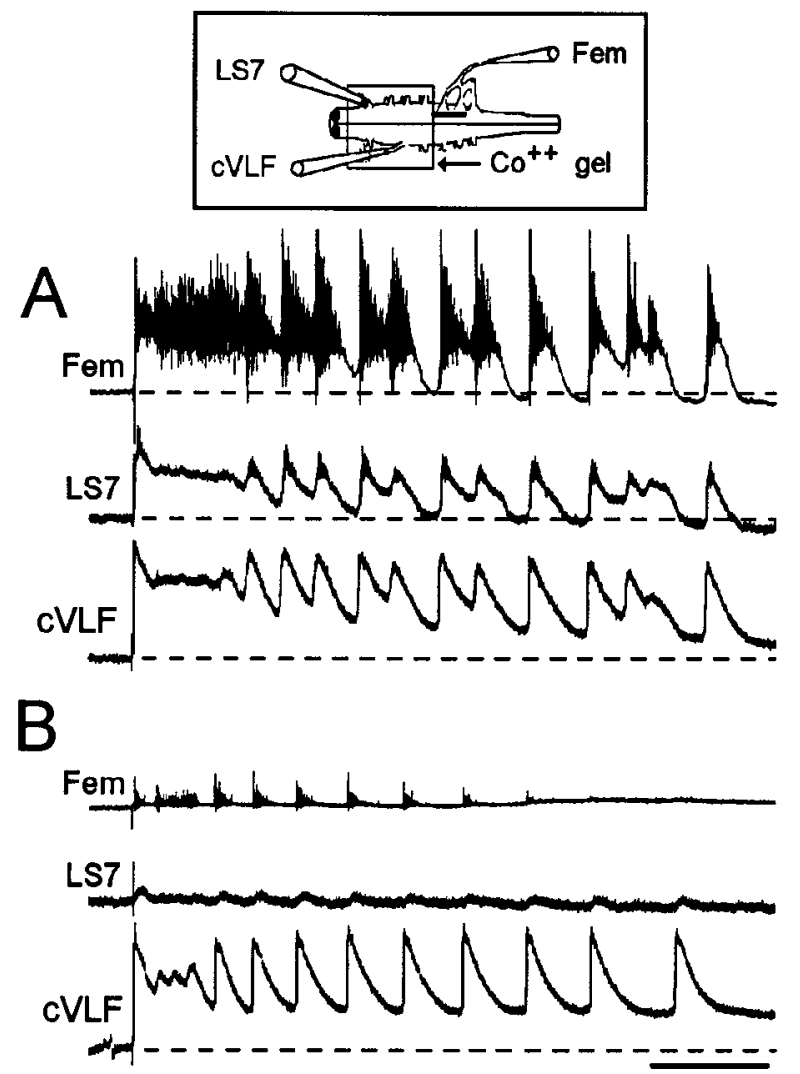

5 sec

Figure 1. A, Recordings from the femorotibialis muscle nerve (Fem), the LS7 ventral root $(L S 7)$, and the contralateral VLF during an evoked episode of rhythmic activity are shown. $B$, A cobalt gel was introduced around the caudal cord (LS3-LS7) to block synaptic transmission. Inset, The recording arrangement is shown. Approximately $30 \mathrm{~min}$ after the gel was applied, the activity of motoneurons under the gel (LS7) was depressed, indicating the efficacy of the blockade. The amplitude of the VLF potentials was slightly increased in the presence of the gel (possibly because of an increase in the space constant of axons within the gel). The decrease of Fem discharge after the gel was applied was probably caused by leakage of $\mathrm{Co}^{2+}$ from the rostral edge of the gel that abuts the femorotibialis pool of motoneurons (black bar on cord in inset). cVLF, Contralateral VLF.

The values for input impedance obtained under the two recording conditions were not significantly different $(p>0.05$, two-tailed Student's $t$ test).

Activity patterns and synaptic drive of single interneurons. Recordings from individual interneurons confirmed the population slow-potential recordings obtained from the VLF. Individual cells received rhythmic, depolarizing synaptic drive in-phase (on the time scale of cycles) with the electrotonically recorded slow potentials recorded from the muscle nerve or the ventral roots (Fig. 2). These electrical recordings confirm previous optical recordings and indicate that interneurons and motoneurons receive synchronized, rhythmic depolarizations during episodes of activity.

The interneurons displayed a range of firing behaviors that could be classified broadly into five groups. These categories are intended as a convenient way of describing a heterogeneous set of cells and are not meant to imply the existence of discrete populations because there was clear continuity between the groups. The identified VLF cells did not constitute a separable subclass; rather their activity was heterogeneous and fell into the same categories as described below for the larger sample of unidentified interneurons. This is not surprising because the number of VLFprojecting neurons may have been underestimated because of failed antidromic invasion.

The first group, which constituted $11.6 \%$ of the total sample and 2 of 10 of the identified VLF cells, comprised cells that did not fire or receive significant synaptic input during rhythmic activity. Some of the cells received small (2-3 mV) synaptic potentials that were not well synchronized with the rhythmic ventral root potentials during episodes of rhythmic activity. Although we cannot exclude the possibility that these cells were damaged, we note that they were neurons because they fired action potentials in response to intracellular current injection.

The second subset of cells $(30.9 \%$ of the sample, four of which were identified VLF cells) received rhythmic drive but did not fire action potentials. These cells did not fire even though their depolarizing synaptic drive could be as large as $35 \mathrm{mV}$ (see Fig. $2 D$ ). Of those cells that fired action potentials during episodes of activity (the third group, $39.8 \%$ of the total sample, including three identified VLF cells), the majority fired irregularly during rhythmic episodes, generating one or two spikes per cycle (Fig. $2 A)$. A minority of cells had discharge patterns that clearly resembled those of motoneurons (groups four and five). One class (5.3\% of the total sample, with one identified VLF cell) fired at the beginning and at the end of each cycle with a pause in firing near the cycle onset (Fig. $2 B$; pauses indicated by arrows). In this respect, these cells resembled sartorius motoneurons that exhibit a similar firing pattern (O’Donovan, 1989). The cells in the final group $(12.4 \%)$ fired more or less continuously during each cycle, accelerating at peak depolarization, thereby resembling extensor motoneurons (Fig. 2C).

Despite the variability in firing, the shape of the underlying synaptic drive in rhythmically active interneurons was similar and resembled that seen in motoneurons (O'Donovan, 1989; Sernagor and O'Donovan, 1991). Generally, it consisted of a depolarization lasting for the duration of the episode, over which was superimposed oscillations corresponding to the individual cycles. The oscillations were synchronized with those of motoneurons, as measured by either the ventral root or muscle nerve potentials (see below for a detailed analysis). The magnitude of the depolarization was highly variable, ranging from a few millivolts to as much as $35 \mathrm{mV}$. However, there was no relationship between the magnitude of the synaptic drive and the resting membrane potential of the cell (Fig. 2D), suggesting that the variability in the amplitude of the rhythmic drive was not simply an artifact of the whole-cell recording (e.g., the result of cellular damage).

To examine the composition of the synaptic drive to interneurons, we established the dependence of the synaptic drive on membrane potential in eight interneurons. This was accomplished by injecting DC current into the cell to change the membrane potential and then evoking an episode by electrical stimulation of the cord surface. In the cell shown in Figure $3 A$, the rhythmic synaptic potentials reversed at approximately -35 $\mathrm{mV}$. The reversal potentials averaged $-35.5 \pm 10.7 \mathrm{mV}$ for eight cells and were scattered over a wide range (Fig. $3 B$ ). We had most success at reversing the nonspiking type of interneuron (six of eight cells), and we could not reverse the rhythmic drive potentials in many other cells, despite holding them at potentials positive to $0 \mathrm{mV}$. The low value for the average reversal potential suggests that the nonspiking types of cells received predominantly depolarizing $\mathrm{GABA}_{\mathrm{a}}$ or glycinergic synaptic inputs during rhythmic activity (see also Sernagor et al., 1995). GABA and 


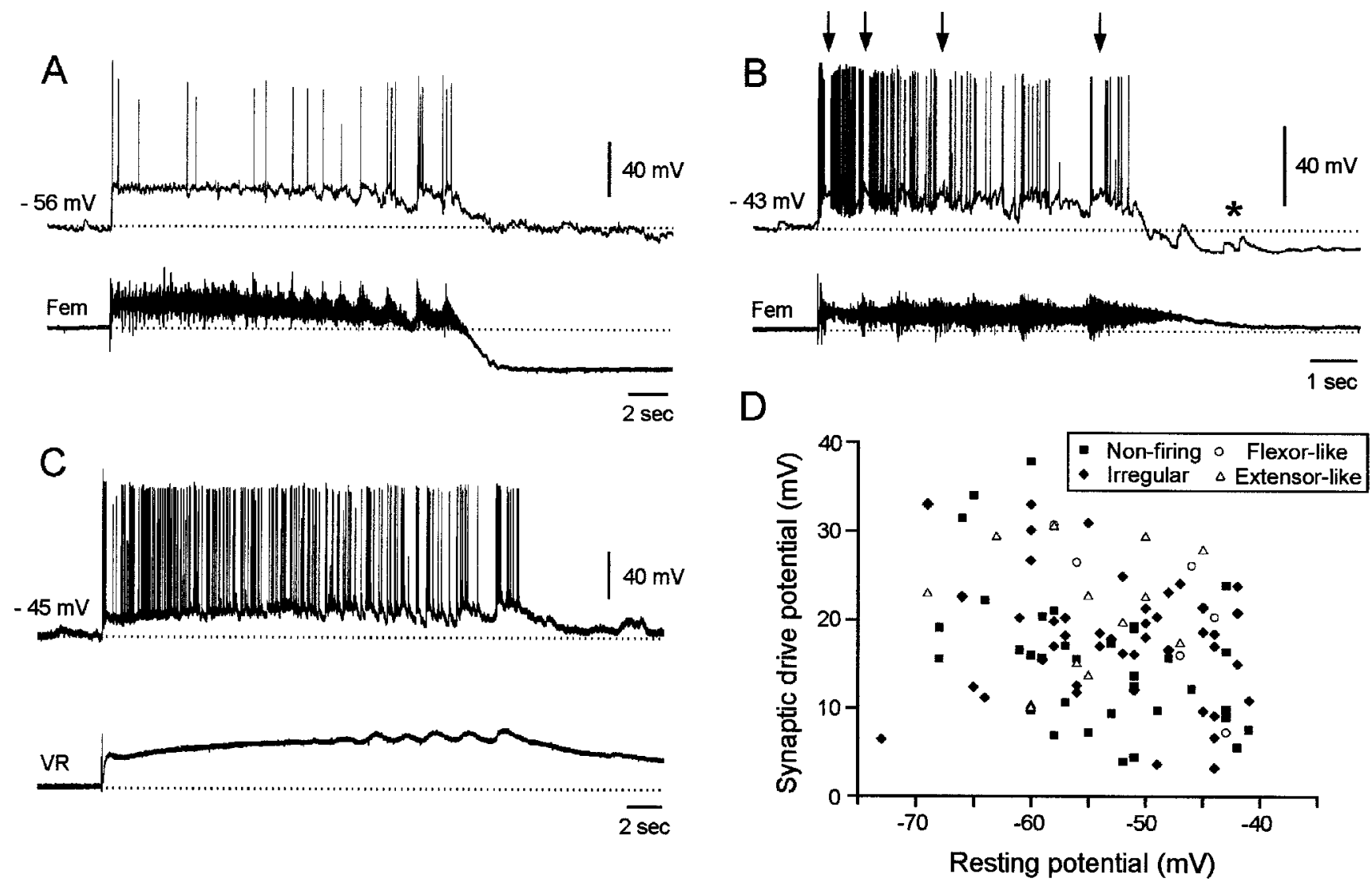

Figure 2. Examples of the activity patterns of ventrally located interneurons during an episode of rhythmic activity. The resting membrane potential is indicated at the beginning of the intracellular record. $A$, Irregularly discharging cell type. $B$, Interneuron with a firing behavior like flexor, sartorius motoneurons. Arrows indicate the pause in each cycle of discharge. The asterisk indicates the postepisode hyperpolarization seen in some cells. $C$, Interneuron with a firing behavior like extensor, femorotibialis motoneurons. $D$, The relationship between resting membrane potential and the amplitude of the peak synaptic drive for a population of 97 ventrally located interneurons. The inset indicates symbols used for the different types of interneuron. $V R$, Ventral root; fem, femorotibialis muscle nerve.

glycine, although depolarizing, can shunt the membrane and prevent action potential generation (O'Donovan, 1989; Sernagor et al., 1995).

Are interneurons coupled by neurobiotin-permeable gap junctions? One mechanism to account for the synchrony in the firing of interneurons and motoneurons might be electrical coupling. Although we did not examine this idea directly - by paired intracellular recordings - we did not test for dye-coupling that is often associated with electrical coupling. For this purpose, we injected the small tracer neurobiotin into individual neurons. With one exception, only single cells were recovered after neurobiotin filling, and in that particular instance we could not exclude the possibility of neurobiotin leakage from the electrode tip. This finding suggests that interneurons are not extensively coupled by neurobiotin-permeable gap junctions at the developmental stages studied here.

All of the neurobiotin-labeled cells were located dorsomedial to the lateral motor column (Fig. $4 A$ ) where a high proportion of rhythmically active interneurons has been detected using calcium imaging (O’Donovan et al., 1994). Cell sizes ranged from 15 to 35 $\mu \mathrm{m}$, measured along the long axis, and cell body shape varied considerably. The numbers of primary dendrites ranged from two to five, with cells rarely exhibiting the large, complicated dendritic trees characteristic of motoneurons. Occasionally an axon could be traced for several hundred micrometers, often terminating, without extensive ramification, in en passant boutons. Two reconstructed cells are illustrated in Figure 4. The axon of one of the cells (Fig. 4B) ramified within the lateral motor column, suggesting the possibility of direct connections between the interneuron and motoneurons. The other cell (Fig. 4C) was identified as projecting its axon into the VLF.

\section{Timing of activity in motoneurons and interneurons}

If ventral interneurons are responsible for the activation of motoneurons, then they should fire before the onset of motoneuron synaptic potentials at the beginning of a spontaneously occurring episode and during each subsequent cycle of activity. To test this idea, we compared the timing of activity recorded from the VLF, the muscle nerves (or ventral roots), and the intracellularly recorded rhythmic synaptic potentials of single interneurons.

Timing of rhythmic activity recorded from muscle nerves, ventral roots, and the VLF

We first compared the population activity recorded from the VLF and muscle nerves. Spike activity was often difficult to resolve in the VLF recordings, presumably because of shunting attributable to the presence of a large number of cut, inactive axons. Nonetheless, in some experiments it was possible to extract the discharge by high-pass filtering $(100 \mathrm{~Hz}$ to $1-3 \mathrm{kHz})$ and then amplifying the VLF signal. Generally, the VLF was dissected 


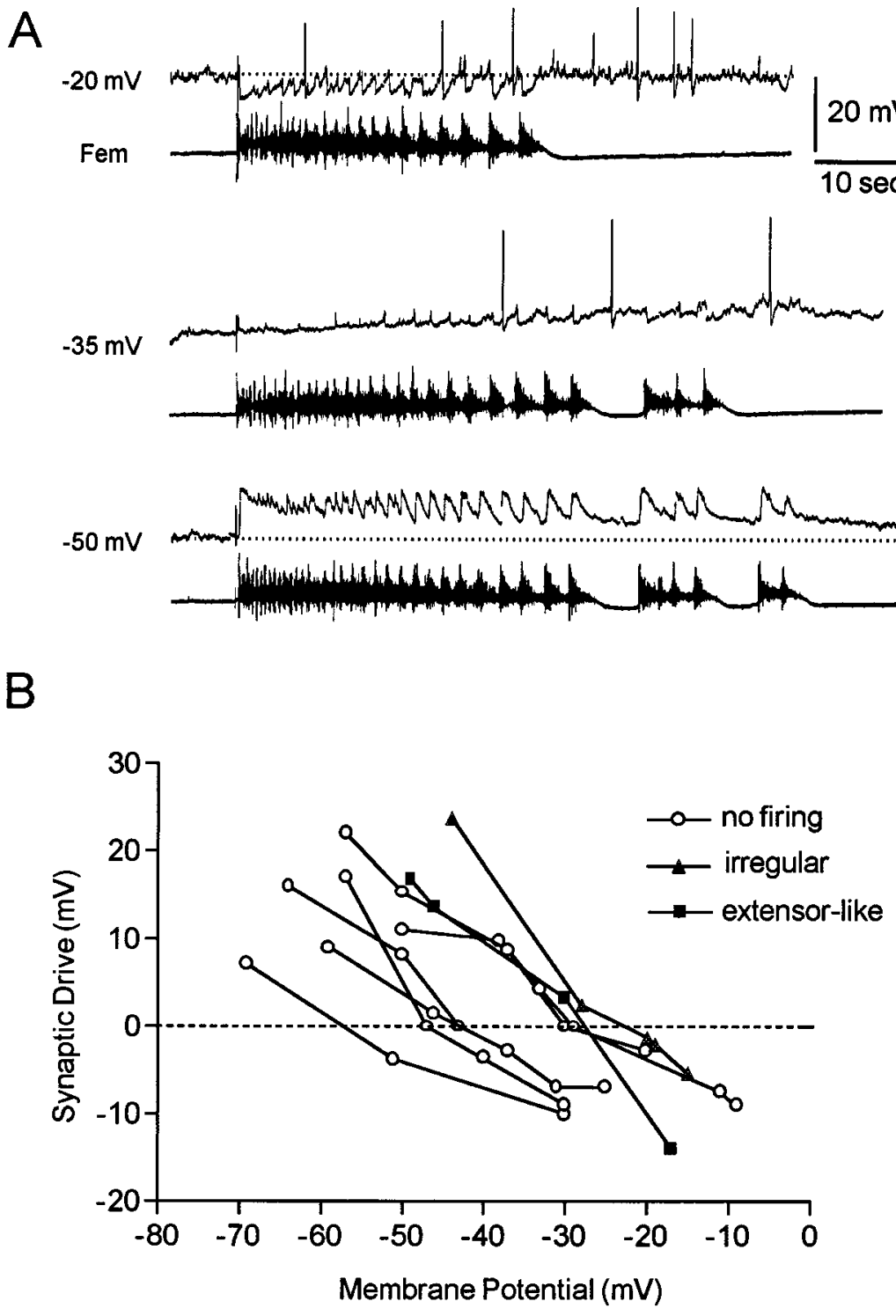

Figure 3. A, The effect of DC current injection on the synaptic drive of a ventrally located interneuron that received rhythmic synaptic potentials but did not fire at the resting membrane potential $(-50 \mathrm{mV})$. The rhythmic drive potentials reversed close to $-35 \mathrm{mV}$. $B$, Plot showing the relationship between the peak amplitude of the rhythmic synaptic drive and membrane potential for eight interneurons. such that it sampled interneurons no less than two segments from the motoneurons that were being recorded. In three experiments, we restricted the contribution of interneurons to the VLF to a single segment (T7) to minimize any delays that might be introduced by sampling interneurons remotely from motoneurons or over several segments of the cord. This was accomplished by dissecting the VLF away from T7 and sectioning it rostrally at the T7-T6 boundary and caudally at the T7-LS1 boundary. We found, however, that such isolation did not significantly alter the timing of the VLF potentials with respect to motoneurons. Figure $5 A$ shows recordings of the rectified integrated neurograms from the sartorius and femorotibialis muscle nerve and the rostral VLF (rVLF; isolated at the level of T7). In this and all of the other experiments, discharge in the VLF occurred after the onset of firing in either the femorotibialis or the sartorius muscle nerves and tended to occur later in the cycle as the episode progressed (see the first four cycles of Fig. $5 A$ ).

To examine the timing of activity in more detail, we focused on the events at the beginning of a spontaneous episode. In some recordings, the earliest activity comprised a slow ramp potential that could be recorded in the VLF, the muscle nerves, and the ventral roots and could start up to $1.0 \mathrm{sec}$ before the episode proper (Fig. $5 B$ ). This ramp was usually clearest in the recordings from the sartorius muscle nerve, where it was often accompanied by irregular spiking (see slow ramp marked in Fig. $5 B$ ). The slow ramp was followed by a rapid rise in the slow potentials (see rapid rise marked in Fig. $5 B$ ) coincident with the onset of the episode. The demarcation between the slow ramp and the rapid rise was generally clearest in the VLF and the ventral roots. Discharge in the muscle nerves preceded both the rapid rise in the slow potential and the discharge recorded from the VLF.

We quantified the timing of the slow potentials and discharge in the muscle nerves by measuring their onset with respect to the onset of the rapid rise in the VLF slow potential (measured at $10 \%$ of the maximum VLF amplitude; 12 episodes in four embryos). The slow ramp in the rVLF and in the muscle nerves began well before the rapid rise in the VLF potential (VLF, $-507 \pm 106 \mathrm{msec}$; sartorius, $-650 \pm 150 \mathrm{msec}$; and femorotibialis, $-514 \pm 97 \mathrm{msec}$ ). The onset of discharge in the rostral VLF was approximately coincident with the time to reach $10 \%$ of the peak amplitude of the VLF $(5 \pm 12 \mathrm{msec}$; measured in eight episodes from three embryos). However, the discharge in the 


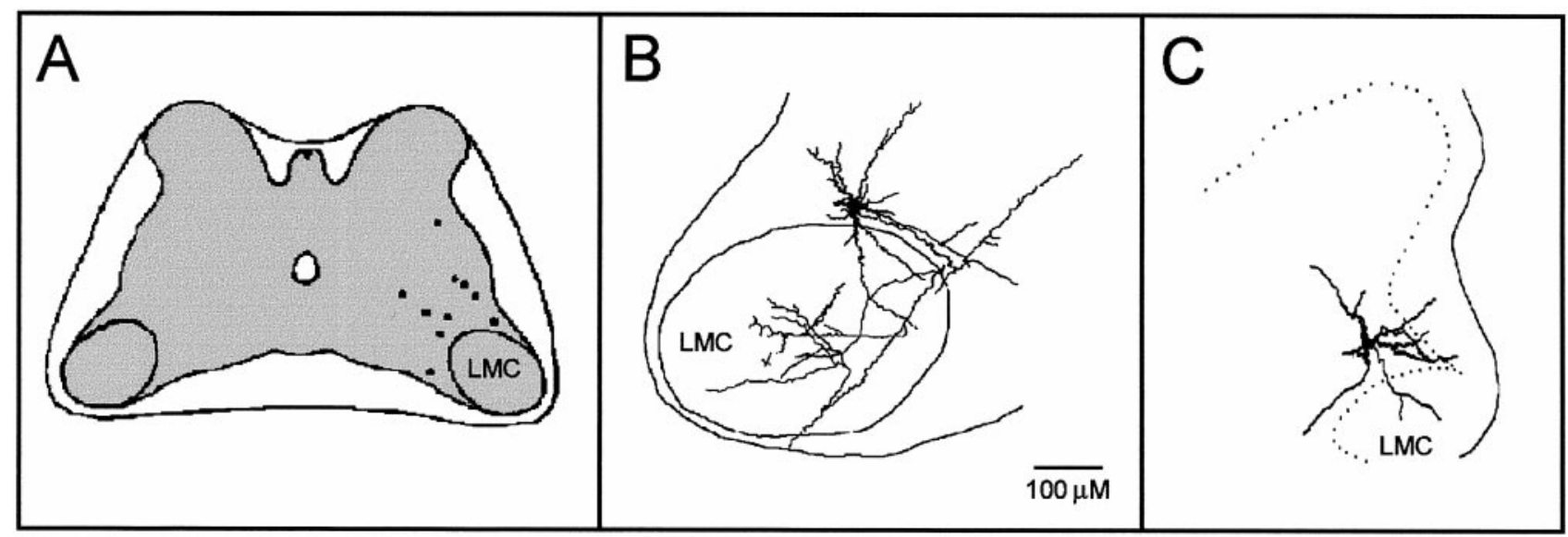

Figure 4. A, Distribution of interneurons recovered after filling with neurobiotin. The lateral motor column $(L M C)$ is indicated in $A-C$. B, Morphology of a ventrally located lumbosacral interneuron with an extensive axonal arborization within the lateral motor column. $C$, Morphology and location of a ventrally located interneuron identified by antidromic activation from the VLF. The dotted line demarcates the gray-white matter boundary.
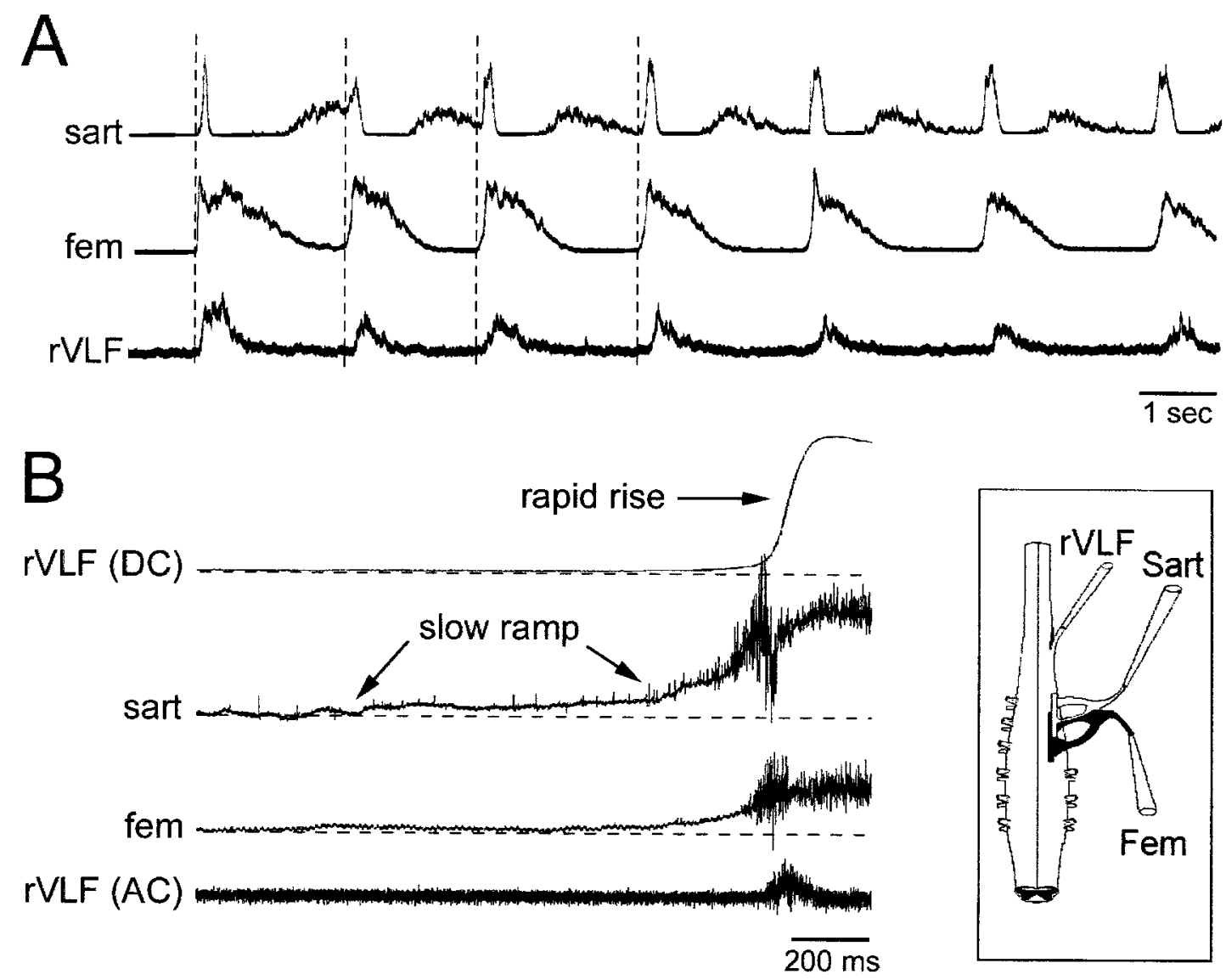

Figure 5. A, Comparison of the timing of discharge in muscle nerves [sartorius (sart) and femorotibialis $($ fem $)$ ] and the rostral VLF $(r V L F)$ during a spontaneous episode of activity. The discharge was filtered at $100 \mathrm{~Hz}$ to $3 \mathrm{kHz}$, rectified, and integrated $(\tau=20 \mathrm{msec})$. The dotted lines are aligned to the onset of discharge in the femorotibialis muscle nerve. $B$, Timing of slow-potential activity recorded from muscle nerves and the VLF at the onset of a spontaneous episode. Inset, The recording arrangement and the approximate location of the sartorius (open vertical bar) and femorotibialis (black vertical bar) motoneuron pools. The VLF slow potentials $[r V L F(D C)]$ were recorded with a bandwidth of $D C$ to $3 \mathrm{kHz}$, and the $r V L F$ discharge $[r V L F$ $(A C)$ ] was recorded with a bandwidth of 100 to $3 \mathrm{kHz}$ and further amplified. The muscle nerve recordings were obtained at $D C$ to $3 \mathrm{kHz}$. The rapid rise of the VLF potential at the onset of the episode and the slow ramp in the sartorius record are indicated by arrows.

sartorius and the femorotibialis muscle nerves began before the rapid rise in the VLF (sartorius, $-87 \pm 18 \mathrm{msec}$, and femorotibialis, $-30 \pm 14 \mathrm{msec})$.

The timing of the slow potentials in the VLF and the muscle nerve was similar in other cycles within the episode. These cycles were most easily measured toward the end of the episode when their onset was clear. In these late cycles the slow potential in the femorotibialis muscle nerve started to rise $65 \pm 18 \mathrm{msec}$ before 

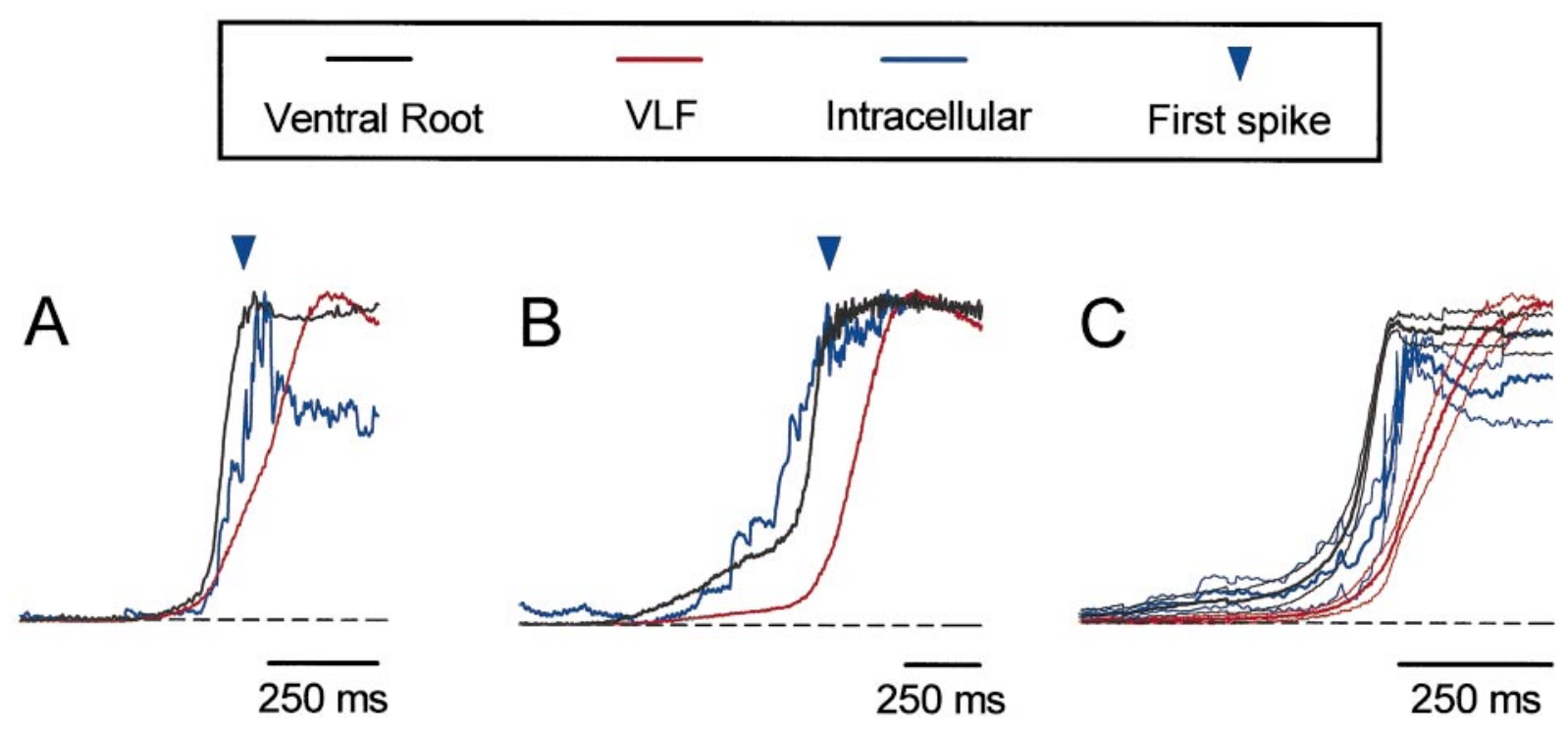

Figure 6. $A, B$, Comparison of the timing of the slow potentials recorded in the VLF and the ventral root with the intracellularly recorded membrane potential of two ventrally located interneurons $(A, B)$ at the onset of a spontaneous episode of rhythmic activity. The arrowheads identify the time of the first spike in the intracellular recording. $C$, An averaged set of traces (5 interneurons) synchronized to the peak ventral root activity. The thick line of each color is the mean, and the thin lines are the SEM.

the onset of activity in the rostral VLF (measured in three late cycles from three episodes in one embryo).

To confirm these population recordings from the VLF and muscle nerves, we compared the onset of intracellularly recorded synaptic potentials in individual interneurons with the slow potentials from the ventral roots and the VLF. We made two types of comparison. First, we compared the onset of the intracellularly recorded rhythmic drive in nine interneurons with the slow potentials recorded from the ventral roots and the VLF (Fig. 6). Secondly, we computed cross-correlograms of the rhythmic intracellular potentials recorded from a further seven interneurons with the slow potentials recorded simultaneously from the femorotibialis muscle nerve and the VLF (Fig. 7).

Comparison of the onset of activity in individual interneurons, the ventral roots, and the VLF

The earliest activity in the interneurons was an increase in the synaptic noise just before the occurrence of the episode. This was followed by a rapid depolarization at episode onset that began after the rapid rise of the ventral root potential in four of five cells but before the rapid rise of the VLF potential (six of six cells). These relationships are illustrated for two interneurons in Figure 6, $A$ and $B$, and are summarized in the averages shown in Figure $6 C$. Quantification of the timing of the intracellular potentials was difficult because of the synaptic noise in the intracellular traces. Therefore, we measured the onset of the first interneuron spike (Fig. 6A, $B$, arrowheads) with respect to the onset of activity in the ventral roots. The first interneuronal spike occurred $474 \pm 171$ msec after the onset of the slow ventral root potential $(n=10$ interneurons; minimum $=121 \mathrm{msec}$; maximum $=1920 \mathrm{msec}$ ). The timing of this initial discharge was coincident with the rapidly rising phase of the VLF slow potential and was, therefore, similar to the timing of the extracellularly recorded VLF discharge.

To provide an estimate of the population behavior from this limited sample of intracellular recordings, we averaged the intracellular potentials from five interneurons synchronized to the peak ventral root activity together with the corresponding VLF and ventral root potentials (Fig. $6 C$ ). This analysis revealed that the averaged intracellular potentials began as a slow ramp at the onset of an episode coincident with the slow ramp in the ventral root recording but preceding the VLF slow ramp. However, both the rapid rise and the peak of the intracellular potentials were delayed with respect to the ventral root potential, although they were faster and earlier than the corresponding components of the VLF potential. The finding that a slow ramp in the intracellular record results from the summation of asynchronous synaptic events in individual cells suggests that the slow ramp recorded in the population recordings of the ventral roots and the VLF may also be generated by this process.

The finding that the rise time and peak of the VLF potentials were delayed with respect to the intracellular recordings is most probably accounted for by electrotonic slowing of the potentials along the VLF axons. By contrast, the timing of spiking recorded intracellularly and from the VLF was similar, as would be expected because the conduction delays for propagated spikes are very short $(\sim 5 \mathrm{msec})$.

Cross-correlation analysis of interneuron and slow-potential activity during an episode of rhythmic activity

In the next set of experiments, we wanted to establish whether the timing we had observed at the onset of an episode was preserved in other cycles within the episode. For this purpose, we crosscorrelated the slow-potential activity of the muscle nerve during an episode of activity with the slow potentials recorded from the VLF ( $n=5$ experiments) and the intracellularly recorded rhythmic drive potentials from seven interneurons. We only used nonspiking cells or those that fired infrequently during an episode to minimize distortions of the synaptic drive potential trajectory.

In five of five experiments, this analysis confirmed that the depolarization of the VLF peaked after that in the femorotibialis muscle nerve (Fig. 7). All five experiments revealed negative lags (mean $=-128 \pm 40 \mathrm{msec}$; range, -40 to $-242 \mathrm{msec}$ ) in the peak of the cross-correlograms between the femorotibialis slow potential and the VLF. By contrast, the correlations between the 

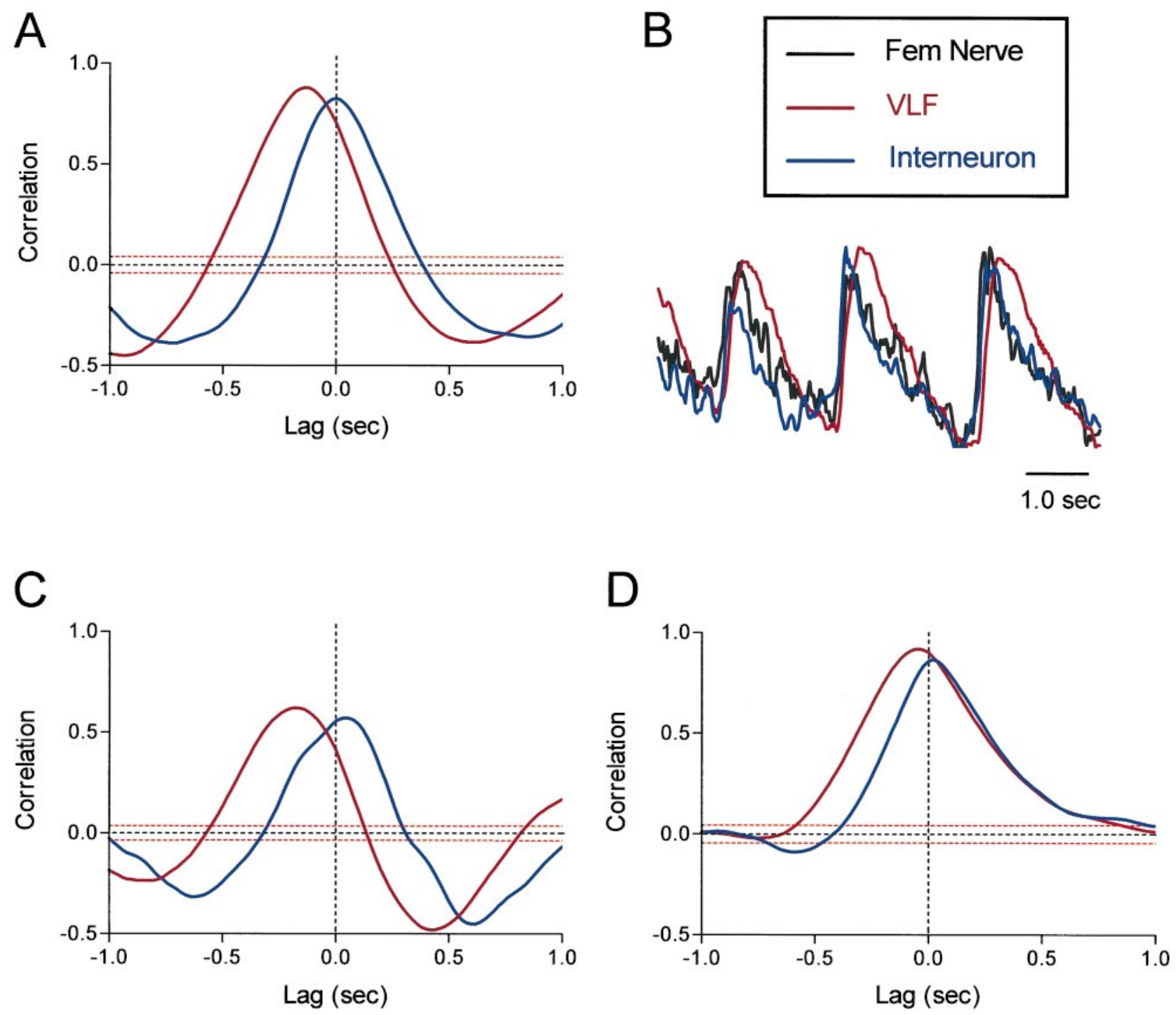

Figure 7. Cross-correlograms of the femorotibialis muscle nerve with the intracellularly recorded interneuronal rhythmic drive potential and population potentials recorded from the VLF. The red dashed lines indicate 3 SEM. $A, C, D$, Correlograms are illustrated for three different neurons. $B$, The traces show the last three cycles of the potentials recorded during an episode of activity that were used to generate the cross-correlogram in $A$.

muscle nerve activity and the intracellular recording indicated that the rhythmic drive potentials tended to peak just after the muscle nerve activity, although some interneurons peaked before the slow potential in the muscle nerve and some after it. The average lag for the peak activity in the cross-correlogram between the femorotibialis muscle nerve activity and the interneuron membrane potential was $-30 \pm 23 \mathrm{msec}$ (range, -110 to +40 msec).

These findings are consistent with the recordings made at episode onset and suggest that the temporal relationships between motoneuron and interneuron activity are similar in each cycle of the episode. Taken together, these findings raise the possibility that motoneurons themselves are important in triggering or reinforcing rhythmic activity. One way that motoneurons could trigger interneuronal activity is through their recurrent connections with interneurons. In previous work, we have shown that motoneurons project directly to a population of spinal interneurons sharing many of the characteristics of the mammalian "Renshaw" cell (O’Donovan et al., 1998b; Wenner et al., 1998).
We have also shown that ventral root stimulation can trigger rhythmic activity apparently via activation of this population (Wenner et al., 1998). If this pathway mediates interneuronal activation, then blockade of recurrent motoneuron collaterals should prevent or significantly alter the recruitment of the VLF and other interneurons.

To test this idea, we compared the timing of VLF and ventral root (or muscle nerve) activity before and after bath application of the nicotinic cholinergic antagonist mecamylamine $(50 \mu \mathrm{M}$; six episodes in two experiments) or a combination of nicotinic cholinergic, muscarinic cholinergic, and calcitonin gene-related peptide (CGRP) antagonists [mecamylamine (50 $\mu \mathrm{M})$, atropine (2 $\mu \mathrm{M})$, and human $\alpha$-CGRP fragment 8-37 (1 $\mu \mathrm{M})$; nine episodes in three experiments; see Fig. 8]. We used antagonists to CGRP because this peptide has been postulated to be released from embryonic chick motoneurons (Carr and Wenner, 1998). We found that spontaneous VLF and motoneuron activity persisted in the presence of either set of drugs (Fig. $8 A$ ), even though the potentials recorded in the ventral roots in response to ventral root 

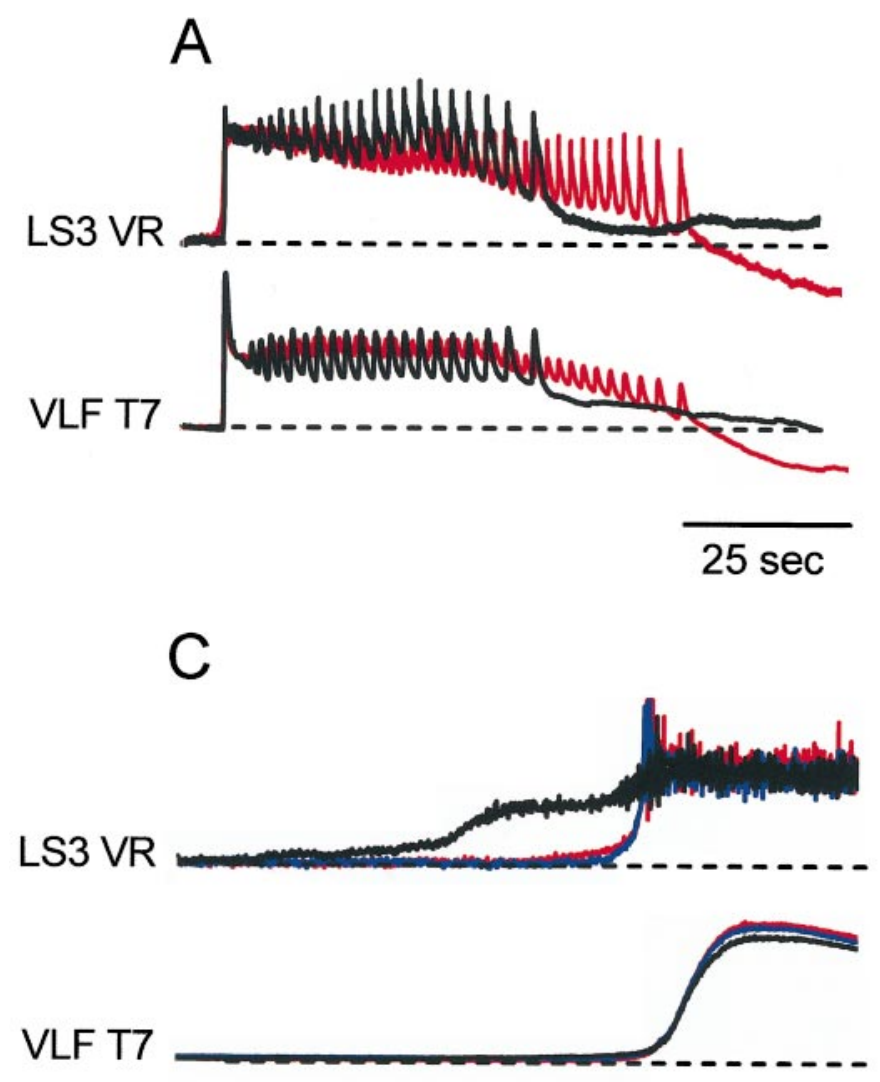

B
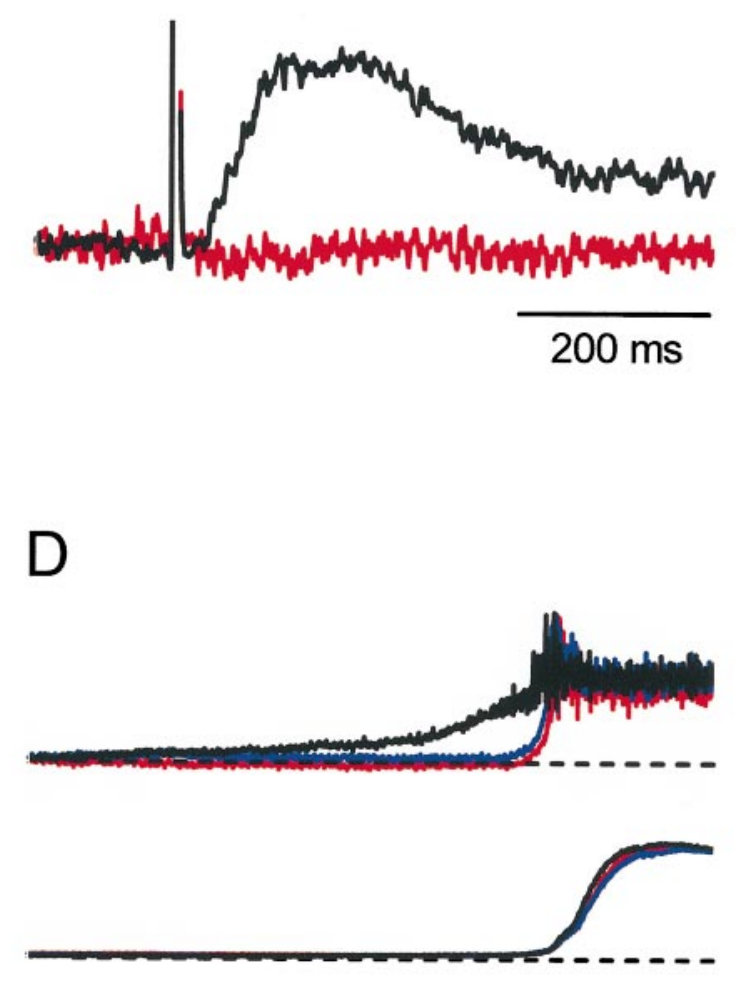

$\overline{250 \mathrm{~ms}}$

Figure 8. Comparison of spontaneously occurring episodes recorded from LS3 ventral root (LS3 VR) and the VLF (VLF T7) before and after bath application of drugs [mecamylamine $(50 \mu \mathrm{M})$; atropine $(2 \mu \mathrm{M})$; and the $8-37$ fragment of human $\alpha$-CGRP $(1 \mu \mathrm{M})$ ] to block the synaptic action of recurrent motoneuron collaterals. A, Comparison of spontaneously occurring episodes before and after application of the drugs (control, black line; drugs, red line). $B$, Recording of the synaptic potentials in the LS3 ventral root after stimulation of the LS2 ventral root before (black line) and after (red line) application of the drugs. $C, D$, The effects of the drugs on the timing of VLF and ventral root activity at the start of spontaneously occurring episodes. The recordings in $C$ were made before the drugs were applied and show three successive episodes (black, red, and blue lines) superimposed. The recordings in $D$ were made after application of the blockers and show three successive episodes superimposed.

stimulation (mediated by recurrent motoneuron collaterals) were blocked (Fig. $8 B$ ). The timing of VLF and motoneuron activity was not altered in two of two experiments in which mecamylamine was used alone. Under control conditions, the peak VLF potential occurred $166 \pm 14 \mathrm{msec}$ (measured in six episodes from two experiments) after the peak ventral root potential, whereas the $10 \%$ rise time of the VLF occurred $36 \pm 15$ msec before the peak ventral root potential. After mecamylamine $(50 \mu \mathrm{M})$, the corresponding values were $161 \pm 6 \mathrm{msec}$ (peak VLF potential) and $-45 \pm 16 \mathrm{msec}(10 \%$ rise time). Application of mecamylamine together with atropine and the CGRP antagonist ( $n=3$ experiments) reduced the amplitude of the potentials and slightly delayed (by $\sim 20 \%$ ) the time to peak of the VLF potentials with respect to those in the motor nerves. The VLF potentials were measured with respect to those recorded from the sartorius muscle nerve in two experiments and the ventral root (LS3) in one experiment. Under control conditions, the peak VLF potential occurred $334 \pm 54 \mathrm{msec}$ (measured in nine episodes from three experiments) after the peak potential in the sartorius muscle nerve or the ventral root. The $10 \%$ rise time of the VLF occurred approximately synchronously $(-4.7 \pm 37$ msec) with the peak in the motor nerves. After the drugs, the corresponding values were $418 \pm 80 \mathrm{msec}$ (peak VLF potential) and $-36 \pm 36 \mathrm{msec}(10 \%$ rise time). After the drugs, the peak amplitude of the motor nerve and the VLF potentials at the onset of the episode was reduced to $77.4 \pm 6$ and $68.9 \pm 13 \%$ of the predrug control responses, respectively (Fig. $8 C, D$ ). These findings indicate that the synaptic activity of recurrent motoneuron collaterals is not required for the occurrence of VLF activity. However, a combination of nicotinic, muscarinic cholinergic, and CGRP blockade depresses VLF and motoneuron activity and slightly delays the time to peak of the VLF potentials.

\section{Synaptic potentials evoked in the ventral roots and interneurons by stimulation of the VLF}

One of the goals of this study was to determine the synaptic organization of premotor networks to establish a mechanism for the synchronous activation of spinal neurons during spontaneous activity. For this reason, we examined the synaptic potentials evoked in motoneurons and interneurons by stimulation of the VLF. We attempted to distinguish the contribution of short (less than five segments) and long (more than five segments) VLF axons to the evoked responses in motoneurons. This was accomplished by comparing the responses evoked in motoneurons when the VLF was stimulated at several different levels of the spinal cord and is described in a later section. 

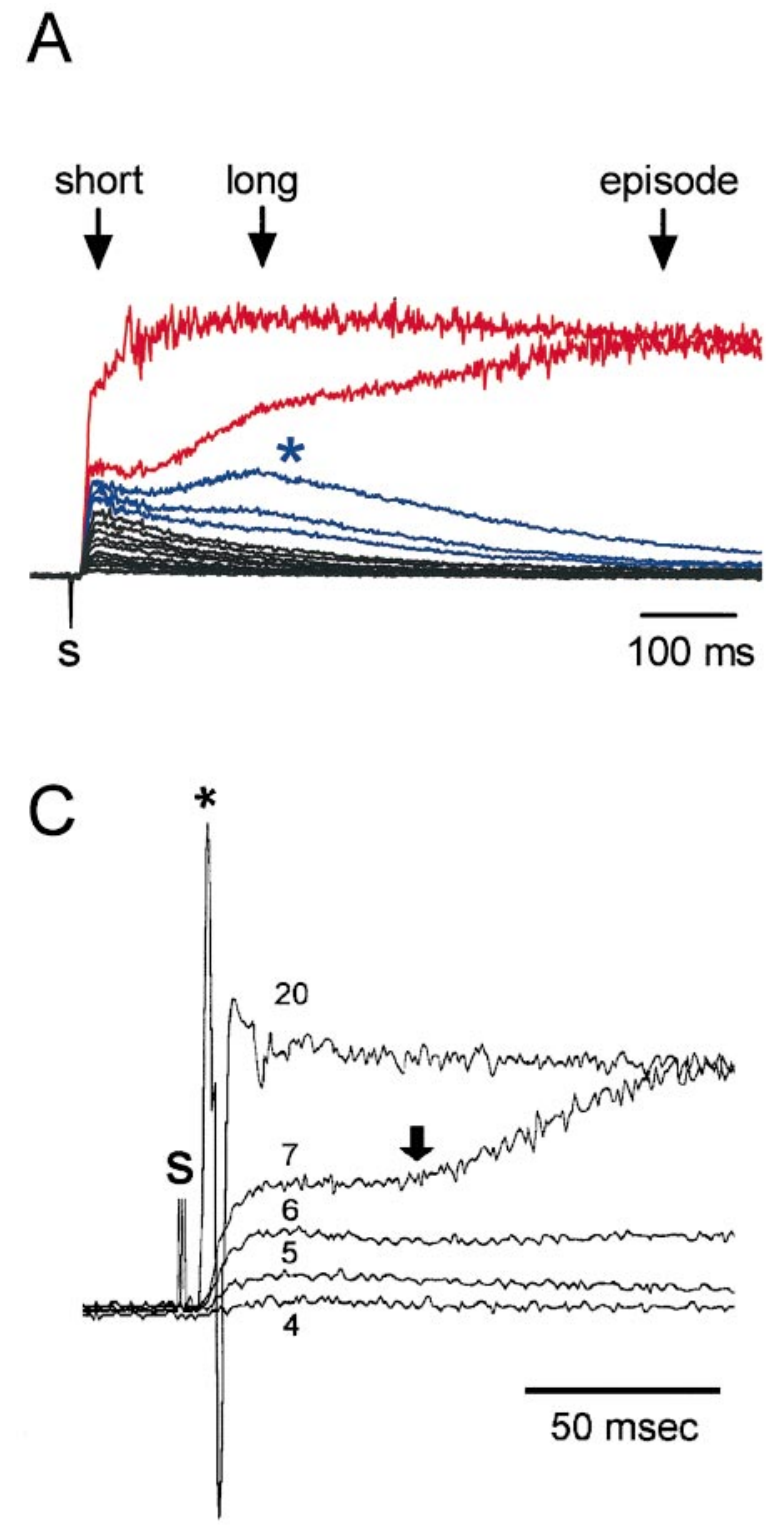
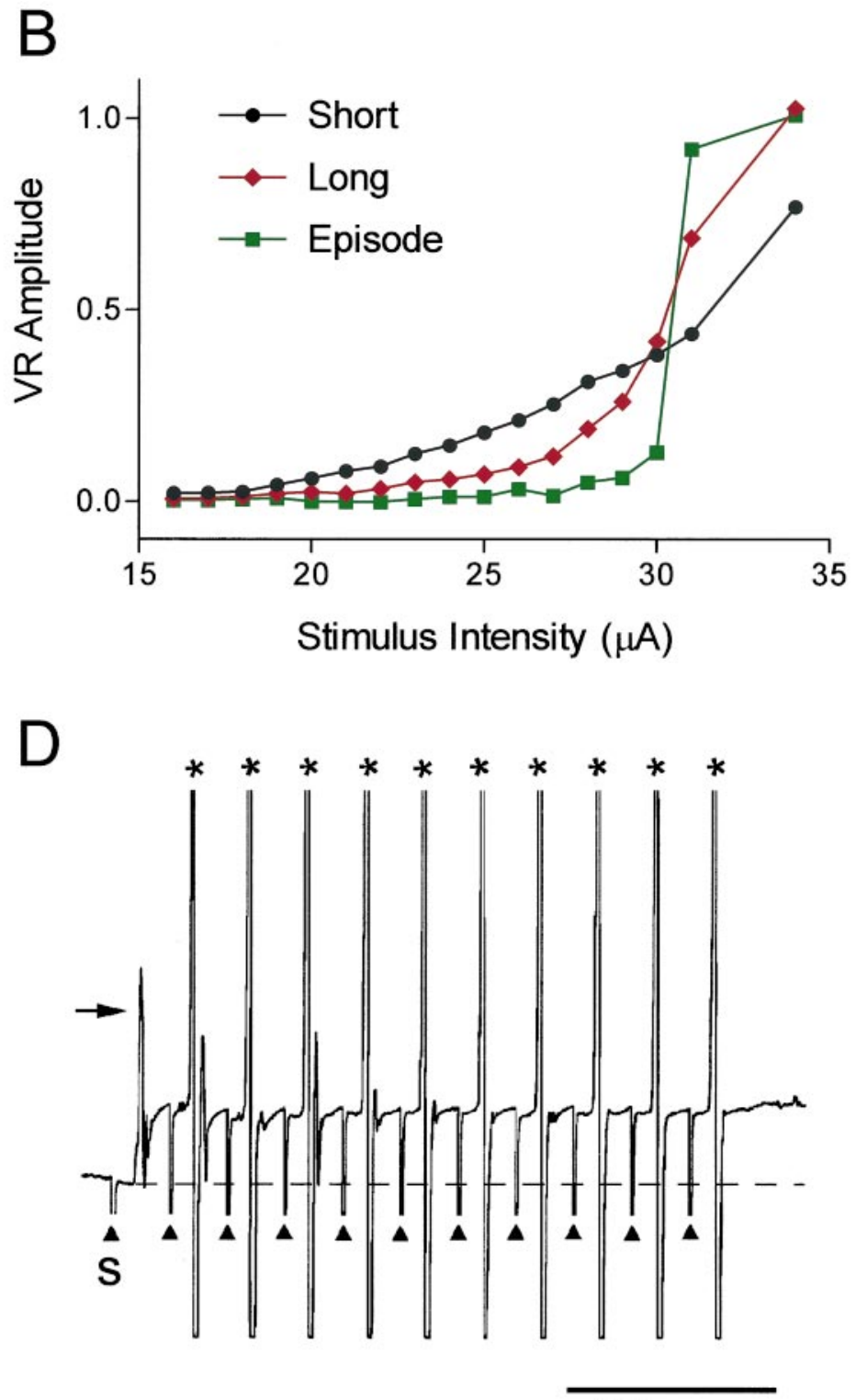

$100 \mathrm{msec}$

Figure 9. $A$, The effects of graded VLF stimulation on evoked ventral root potentials are shown. The current intensity was increased from 16 to $31 \mu \mathrm{A}$ in $1 \mu \mathrm{A}$ increments. The arrows indicate when amplitude measurements were made on the short- and long-latency components and after an episode had been triggered (episode). The blue asterisk indicates the appearance of the long-latency component in the blue traces. The red traces indicate activation of an episode. $B$, Plot of the normalized amplitude of the three components (short, long, and episode) as a function of the stimulus current is shown. $C$, Potentials are recorded from the sartorius muscle nerve in response to graded stimulation of the VLF. The numbers on the individual traces indicate the stimulus current (in microamperes), and $S$ indicates the stimulus artifact. In this example, the VLF was stimulated suprathreshold for triggering of an episode $(20 \mu \mathrm{A}$; episode threshold, $7 \mu \mathrm{A}$, indicated by an arrow) and evoked a brief, high-intensity discharge in the muscle nerve (referred to as the synchronous spike; marked by an asterisk). $D$, The synchronous spike evoked in motoneurons was resistant to high-frequency ( $30 \mathrm{~Hz}$ ) stimulation. The horizontal arrow identifies the first synchronous spike. The first stimulus artifact is marked by $S$ and an arrowhead. Subsequent artifacts are indicated by arrowheads.

\section{Ventral root and VLF potentials evoked by VLF stimulation}

Figure $9 A$ illustrates the potentials recorded in the ventral roots in response to a progressive increase in the stimulus current applied to the VLF. In this experiment, a short-latency synaptic potential (onset 9 msec after the stimulus) was activated at the lowest stimulus intensity $(16 \mu \mathrm{A})$. The amplitude of this potential grew with increasing stimulus current until $28 \mu \mathrm{A}$ when a distinct longer latency component first became evident (Fig. 9A, asterisk over blue traces). Very small further increases in stimulus current resulted in nonlinear growth of the long-latency component so that by $31 \mu \mathrm{A}$ an episode was triggered. Similar findings were made in three other experiments in which graded stimulation of the VLF was used (see Fig. 9C). The ventral root potentials evoked by VLF stimulation were generated by chemical synapses because they were reversibly abolished by $10 \mathrm{mM} \mathrm{Co}^{2+}(n=3$ experiments; data not shown).

The recruitment of the ventral root potentials by VLF stimulation is quantified in Figure $9 B$. The short-latency component (Fig. 9B, black curve; measured $\sim 25 \mathrm{msec}$ after the stimulus) exhibited an approximately linear relationship to the stimulus 
current until the stimulus was just suprathreshold for triggering an episode $(31 \mu \mathrm{A})$. By contrast, the long-latency responses (Fig. $9 B$, brown curve; measured $\sim 200$ msec after stimulus) initially rose linearly with stimulus intensity (up to $\sim 25 \mu \mathrm{A}$ ) but then rose more rapidly until an episode was triggered. After an episode was triggered, the long-latency component merged into the depolarization associated with the episode (Fig. 9A, lower red trace). When just subthreshold for episode activation, the long-latency responses decayed slowly to the baseline over $500 \mathrm{msec}$. The graph for recruitment at $\sim 610 \mathrm{msec}$ (Fig. 9B, green curve, labeled episode) was made when the short- and long-latency synaptic responses had almost completely decayed and revealed the abrupt threshold for episode recruitment at $31 \mu \mathrm{A}$. Further increases in stimulus intensity shortened the latency of episode recruitment but did not alter the amplitude of the ventral root potential, suggesting that the output of the network had saturated. This nonlinear recruitment pattern of the long-latency component suggests a regenerative mechanism within the network and is considered more fully in the Discussion.

A brief, intense, and short-latency spike could be evoked when the stimulus intensity to the VLF was suprathreshold for an episode (Fig. 9C). This spike-hereafter referred to as the "synchronous spike" - was resistant to fatigue and could sustain stimulation frequencies up to $30 \mathrm{~Hz}$, although with some decrease in its amplitude (Fig. 9D). This behavior is in marked contrast to that of monosynaptic, muscle afferent EPSPs that depress rapidly at stimulation frequencies of $5 \mathrm{~Hz}$ (Lee and O'Donovan, 1991). We assume that the synchronous spike is generated by a shortlatency synchronized discharge of motoneurons in response to the VLF stimulus and is a combination of a propagated compound action potential and electrotonically recorded motoneuron spikes. Its appearance during the episode presumably occurs because the motoneurons are depolarized and therefore suprathreshold for spiking. These responses are probably mediated monosynaptically because of their short latency (see Fig. 9C, asterisk) and their relative resistance to fatigue during repetitive stimulation.

Stimulation of the VLF also evoked slow, population potentials that could be recorded in a portion of the VLF more rostral or caudal to the part we stimulated. When the VLF was stimulated this way, a short-latency volley was recorded that preceded the evoked synaptic potential. This volley probably represented activation of VLF axons running from the stimulating to the recording electrode, but it may also have included synaptic field potentials from the adjacent gray matter. In contrast to motoneuron recordings, VLF stimulation failed to evoke a synchronous spike in the VLF even at stimulus intensities suprathreshold for an episode.

Whole-cell recordings from motoneurons and interneurons confirmed the presence of short- and long-latency connections between the VLF and motoneurons and interneurons (Fig. 10). Although only a small percentage of the sample could be antidromically activated by stimulation of the VLF $(\sim 10 \%)$, nearly all of the remaining cells $(95 \%)$ received synaptic input from the VLF. The amplitude of the VLF-evoked PSPs averaged $6.3 \pm 5.7$ $\mathrm{mV}$ with an average latency of $8.9 \pm 5.0 \mathrm{msec}$. Nearly one-half the sample $(47 \%)$ had latencies of $10 \mathrm{msec}$ or less. The short latency of these potentials suggests that the earliest components are mediated monosynaptically. Although latencies can be difficult to interpret, monosynaptic muscle afferent EPSPs evoked in motoneurons (over a similar conduction distance) have an average

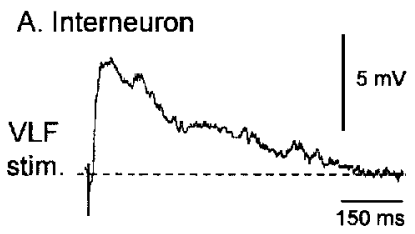

B. Interneuron
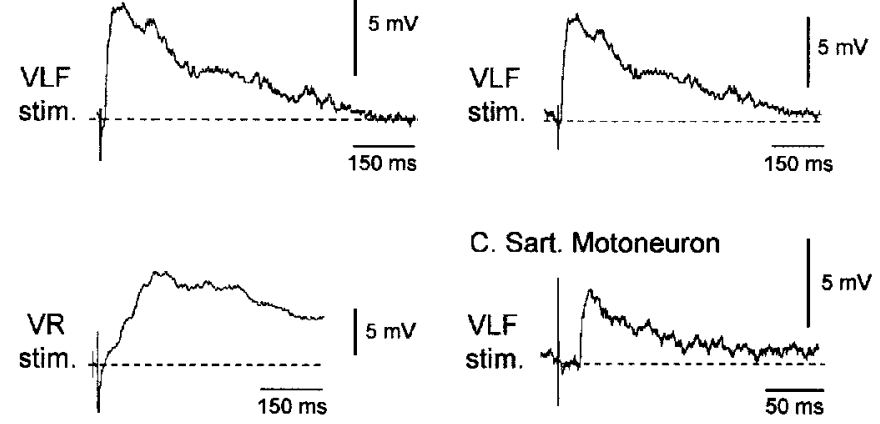

Figure 10. Synaptic potentials evoked in individual interneurons in response to VLF stimulation. $A$, Synaptic potentials recorded in a ventrally located interneuron in response to VLF (upper trace) and ventral root (lower trace) stimulation. Notice that the interneuron is depolarized by the ventral root stimulus presumably by synaptic release of acetylcholine from recurrent motoneuron collaterals. Consistent with this interpretation, these synaptic potentials are blocked by mecamylamine (P. Wenner and M. J. O'Donovan, unpublished observations). B, VLF-evoked intracellular potential recorded in another ventrally located interneuron located in LS2. $C$, VLF-evoked synaptic potential recorded in a sartorius motoneuron. stim., Stimulation.

latency of $10 \mathrm{msec}$, whereas polysynaptic EPSPs have a minimum latency of $16 \mathrm{msec}$ (Lee and O'Donovan, 1991).

\section{Origin of axons giving rise to VLF-evoked ventral root potentials}

The potentials evoked by VLF stimulation could arise from the activation of descending, ascending, or propriospinal axons traveling in the VLF. To establish the contribution of long-range projections (more than five segments from the recorded segment) to the evoked responses, we activated the VLF sequentially at various levels of the thoracic cord (generally T1, T3 or T4, T5, and T7) and recorded the responses from the femorotibialis (external) muscle nerve that originates from the lumbosacral segments 2 and 3. We also recorded the presynaptic, axonal volley in the VLF using an extracellular glass microelectrode inserted into the VLF near T7 or LS1 (Fig. 11C).

We first stimulated the entire cut end of the cord at T1 to ensure that all of the descending, ascending, and long propriospinal axons at this level were activated. As illustrated in Figure $11, A$ and $B$, stimulation at this level did not produce a shortlatency synchronized spike in motoneurons or a significant volley in the VLF at LS1. Similar observations were made when a strip of the VLF was stimulated at T1. When the stimulating electrode was moved caudally to T4, the VLF stimulus evoked a small amplitude spike and a distinct but small axonal volley that followed stimulation at $20 \mathrm{~Hz}$. When the stimulating electrode was moved to T7, the short-latency synchronous spike was recruited, and the axonal volley increased in amplitude dramatically.

The simplest interpretation of these findings is that the number of VLF axons projecting to motoneurons progressively increases as the stimulating electrode is moved closer to the recording site. The amplitude of the VLF volley increased several-fold when the electrode was moved from T4 to T7, suggesting that the number of axons recruited by the VLF stimulus increases correspondingly, although some of this increase may be caused by higher synchrony of the afferent volley because of the reduced conduction distance. This finding, together with the abrupt appearance of the short-latency synchronous spike on moving the electrode 


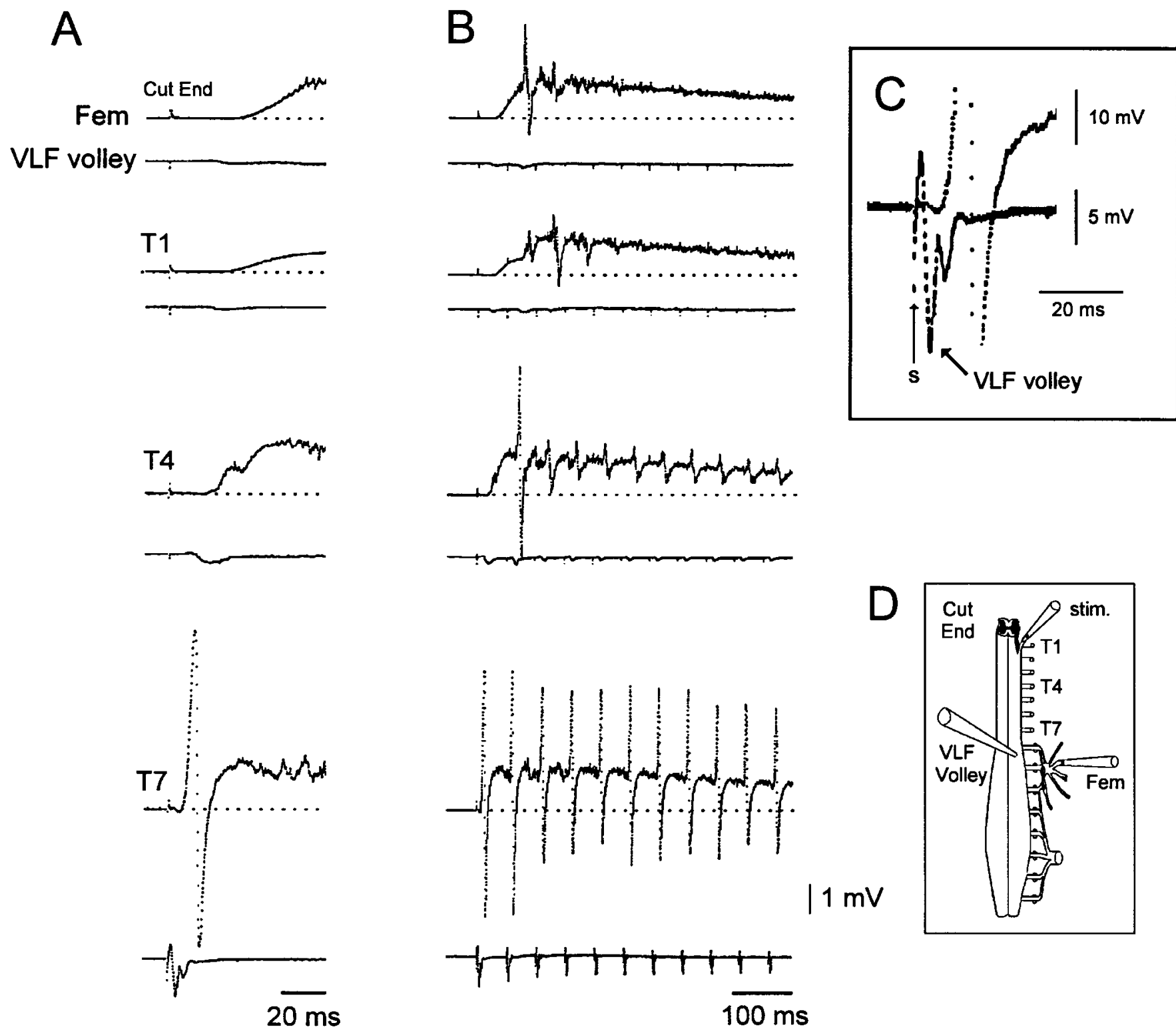

Figure 11. Physiological evidence that the short-latency synchronous spike evoked in motoneurons by VLF stimulation is mediated by short $(<5$ segments) propriospinal rather than descending, ascending, or long-range propriospinal axons. $A$, The spinal cord was stimulated at several different levels (the rostral cut end at T1; the VLF at T1, T4, and T7; see $D$ ). The response in motoneurons was monitored from the femorotibialis muscle nerve $($ Fem $)$ and the afferent volley $(C, D$, VLF volley) recorded by a glass electrode inserted into the VLF near T7 or LS1 ( $D)$. B, Records were obtained during a train at $20 \mathrm{~Hz}$ applied to the VLF. Notice the rapid growth in the amplitude of the synchronous response when the electrode is moved from T4 to T7. $S$, Stimulus artifact.

from $\mathrm{T} 4$ to $\mathrm{T} 7$, suggests that these responses are mediated in part by the activation of propriospinal axons with short projections (less than five segments) to motoneurons.

\section{DISCUSSION}

One of the major goals of this study was to investigate the organization of interneuronal networks responsible for providing rhythmic synaptic drive to motoneurons during spontaneous activity. We found that ventrally located interneurons, including a subset whose axons project in the VLF, were rhythmically active and projected to both interneurons and motoneurons. These data, together with our previous findings (Ho and O'Donovan, 1993; O’Donovan et al., 1994; O'Donovan and Ritter, 1995b), are consistent with the idea that ventrally located interneurons provide some of the depolarizing rhythmic drive to motoneurons during episodes of rhythmic activity.

\section{Role of ventrally located interneurons in triggering episodes of rhythmic activity}

However, given this conclusion, we made the surprising observation that the discharge of the VLF or individual interneurons occurred after the onset of synaptic potentials in the ventral roots or muscle nerves. This finding clearly raises the possibility that motoneurons might be involved in the initiation and perhaps also in the maintenance of spontaneous activity by developing spinal networks. Such a role has been demonstrated previously for spinal motoneurons in episodes of swimming in the embryonic tadpole (Perrins and Roberts, 1995). 
However, several observations argue against a substantial role for motoneurons in episode initiation or as essential components for the expression of spontaneous activity. Most importantly, spontaneous episodes of rhythmic VLF and motoneuron activity persisted after blockade of recurrent motoneuron collaterals with a combination of antagonists. In the presence of nicotinic and muscarinic cholinergic antagonists together with the 8-37 fragment of human $\alpha$-CGRP to block the actions of the peptide CGRP (Carr and Wenner, 1998), a small decrease in the amplitude and time to peak of the VLF potentials was observed. Nonetheless, it is clear that the spontaneous activation of interneurons recorded from the VLF does not require the synaptic action of recurrent motoneuron collaterals.

Other observations indicate that motoneurons are probably not required for the expression of spontaneous activity. For example optical lesioning of motoneurons (O'Donovan and Ritter, 1995b), their selective inactivation by retrograde transport of the $\mathrm{Na}^{+}$ channel blocker QX314 (Wenner and O'Donovan, 1995), or their surgical removal (Wenner and O'Donovan, 1996) all preserve the occurrence of spontaneous activity recorded from the VLF.

Thus, although we cannot definitively exclude the involvement of motoneurons in episode initiation, the arguments we have presented suggest the operation of some other mechanism. The most obvious alternative is that interneuronal activity triggers the episode and drives motoneurons. To understand how this could occur-given the timing of interneuron and motoneuron activity-it is necessary to review the events that take place at the onset of a spontaneous episode of activity. The earliest activity in spinal neurons is an increase in the amplitude of spontaneous miniature synaptic events of which many may be action potential independent (Chub and O'Donovan, 1998). As we have discussed, the slow ramp recorded in the ventral roots and the VLF probably reflects the summation of these asynchronous synaptic events from many cells. Coupled with these changes are a progressive recovery from a prolonged postepisode depression of synaptic transmission in active pathways (Fedirchuk et al., 1999) and the dissipation of a postepisode hyperpolarization of spinal neurons (Chub and O'Donovan, 1995). These events combine to trigger activity in some interneurons that eventually propagates to recruit the whole network (i.e., trigger an episode) via positive feedback (see below).

To account for the timing of motoneuron and interneuron activity, it is necessary to postulate that motoneurons (as a population) are recruited more easily and more rapidly than are interneurons (as a population). This could occur for several reasons. For example, the most excitable or most easily recruitable interneurons might project preferentially to motoneurons and possess weaker connections to the rest of the interneuronal network. Consistent with this idea is the observation that VLF stimulation produced an intense short-latency response (the synchronous response) in motoneurons but not in the VLF during an episode, suggesting that VLF projections are more potent to motoneurons that to other VLF interneurons. Another possibility is that motoneurons possess more NMDA receptors or other voltage-dependent channels than do interneurons. Correspondingly, small synaptic depolarizations could lead to a rapid, regenerative depolarization of the motoneuron membrane.

\section{Network recruitment and synchrony}

A striking feature of the spontaneous activity generated by spinal networks is that all of the constituent neurons are synchronously activated (at the time scale of cycles) during an episode. Indeed, this synchronous pattern of activation is characteristic of developing networks in many parts of the nervous system (see Katz and Shatz, 1996). One of the mechanisms contributing to synchronization in spinal networks is the depolarizing nature of the classically inhibitory neurotransmitters glycine and GABA (Obata et al., 1978; Wu et al., 1992; Sernagor et al., 1995; Nishimaru et al., 1996). Data presented in this paper suggest that another factor may be the synaptic architecture of developing networks, in particular, the existence of recurrent functionally excitatory connections between spinal neurons (see Fig. 12, schematic). We base this conclusion on two pieces of evidence derived from the behavior VLF-evoked potentials recorded in motoneurons and interneurons.

It is difficult to interpret the effects of VLF stimulation because the pathway contains axons from propriospinal, ascending, and descending neurons (Oppenheim et al., 1988; Yaginuma et al., 1990; Webster and Steeves, 1991; Necker, 1992; Pinco and LevTov, 1994; Sholomenko and O'Donovan, 1995). Nevertheless, several lines of evidence suggest that a proportion of the VLF axons stimulated at T5-T7 or LS5-LS6 arise from neurons in the lumbosacral cord that are activated during spontaneous episodes. The strongest evidence of this is indirect but nevertheless persuasive. In other work (Fedirchuk et al., 1999), we have shown that VLF-evoked synaptic potentials in motoneurons and interneurons are depressed by activity, as are other synapses in the developing spinal cord (Lee and O’Donovan, 1991; Lev-Tov and Pinco, 1992). After an episode of spontaneous activity, VLFevoked potentials in motoneurons and interneurons can be depressed to $20 \%$ of their pre-episode amplitude. Our previous work has shown that this depression requires the previous activity of the stimulated axons (Fedirchuk et al., 1999). As such the depression becomes a marker for active, presynaptic neurons. These findings suggest that a significant fraction of the stimulated axons originate from neurons that are firing during the episode. When this inference is combined with the finding that most, if not all, rhythmically active spinal interneurons receive synaptic input from the VLF, we can conclude that rhythmically active interneurons project synaptically to each other.

A second piece of evidence in favor of this pattern of organization comes from the behavior of the VLF-evoked potentials in response to graded stimulation of the VLF. In particular, we found that long-latency VLF-evoked responses exhibited very nonlinear recruitment with increasing stimulus intensity and eventually evolved into an episode. This is unlikely to be caused by a nonlinear increase in the number of stimulated axons because the short-latency VLF-evoked responses grew approximately linearly over the same stimulus increments. We propose that this nonlinear behavior of long-latency responses reflects positive feedback excitation within a network of recurrently connected spinal interneurons. We also suggest that the prolonged duration of the long-latency synaptic responses $(>500 \mathrm{msec})$ is due, in part, to the sequential synaptic activation of recurrently connected neurons.

These ideas are illustrated in the model shown in Figure 12. When the stimulus intensity is high enough to evoke long-latency responses, it does so because enough neurons are activated directly to recruit additional neurons by spatial summation of their output projections. At these stimulus intensities, only partial recruitment of the interneuronal population occurs, and the response dies out (Fig. 12A, middle). With further stimulus increases, enough neurons are recruited initially to trigger positive feedback recruitment of the whole interneuronal population, and 

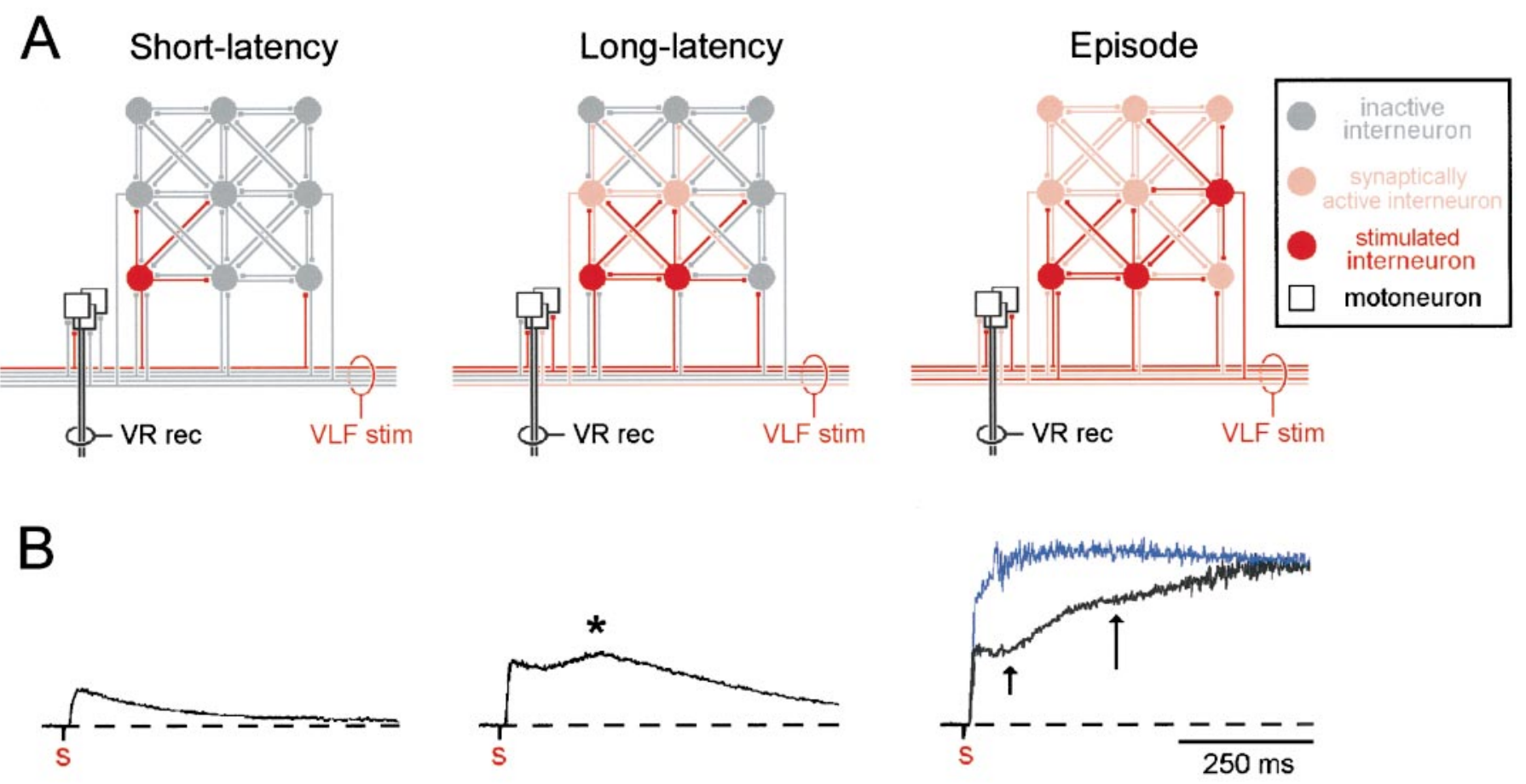

Figure 12. A, Diagrams of the hypothesized synaptic organization and recruitment of VLF interneurons during graded stimulation of the VLF. $B$, Electrical responses recorded from the ventral roots during graded stimulation of the VLF (data from Fig. 9A). Three conditions are illustrated. Left, Low-threshold stimulation evoking only short-latency responses in motoneurons. Middle, An intermediate intensity of stimulation evoking long-latency responses. Right, Suprathreshold stimulation that triggers regenerative recruitment of the whole network and causes an episode to occur. The color code in $A$ illustrates the neurons we hypothesize are recruited under the three stimulus conditions. The VLF is indicated by the parallel lines surrounded by the ellipses. The red lines (and cell bodies) indicate which axons have been activated directly by the stimulus. Notice that the number increases from left to right as the stimulus intensity is increased. Gray lines and cells are not activated directly by the stimulus. At the lowest stimulus intensity, it is assumed that the synaptic responses recorded in the ventral roots (shown in $B$ ) are mediated primarily by direct, monosynaptic contacts between the VLF axons and motoneurons. Notice in $A$, left, that the cell body of one neuron is antidromically activated but that this does not spread to recruit any of the other VLF neurons to which it is connected. At an intermediate intensity of stimulation (middle), several axons are directly activated (red), and these stimulated neurons synaptically activate other neurons (pink). These recurrently connected, synaptically activated neurons underlie the long-latency responses (asterisk). At the highest intensity of stimulation shown (right), three neurons are stimulated directly (red), and this results in regenerative recruitment of the whole network, and an episode occurs. In $B$, right, two stimulus intensities are illustrated. The lowest intensity (black trace) is just suprathreshold for an episode and evokes a long-latency response (at small arrow) that develops into an episode (at long arrow). When the stimulus intensity is well above threshold (blue trace), an episode is triggered without the intervening recruitment of the long-latency response (see text for details). $S$, Stimulus artifact.

an episode is triggered (Fig. $12 \mathrm{~A}$, right). When the stimulus is just adequate to trigger an episode, there will be a delay as activity propagates to recruit the whole network. At higher stimulus intensities, network recruitment occurs without a substantial delay as illustrated in Figure $12 B$ (right).

We hypothesize that this pattern of recurrent, excitatory connectivity is the foundation of spontaneous network recruitment and synchrony in the developing spinal cord (for review, see O'Donovan and Chub, 1997; O’Donovan et al., 1998b; O’Donovan, 1999). A similar network organization may be responsible for spontaneous activity in other parts of the nervous system. At present our evidence of recurrent connectivity is indirect, and in future experiments it will be necessary to document the connections of individual, rhythmically active interneurons to provide proof of this pattern network organization.

\section{REFERENCES}

Aghajanian GK, Rasmussen K (1989) Intracellular studies in the facial nucleus illustrating a simple new method for obtaining viable motoneurones in adult rat brain slices. Synapse 3:331-338.

Carr PA, Wenner P (1998) Calcitonin gene-related peptide: distribution and effects on spontaneous rhythmic activity in embryonic chick spinal cord. Dev Brain Res 106:47-55.

Chub N, O’Donovan MJ (1995) Multi-functional actions of GABA in the regulation of spontaneous activity in the isolated spinal cord of the chick embryo. Soc Neurosci Abstr 21:688.

Chub N, O'Donovan MJ (1998) Miniature postsynaptic currents and their modulation by spontaneous activity in the E11-E12 chick embryo spinal cord. Soc Neurosci Abstr 24:1315

Fedirchuk B, Wenner P, Whelan PJ, Ho S, Tabak J, O'Donovan MJ (1999) Spontaneous network activity transiently depresses synaptic transmission in the embryonic chick spinal cord. J Neurosci, 15: 2102-2112.

Garaschuk O, Hanse E, Konnerth A (1998) Developmental profile and synaptic origin of early network oscillations in the CA1 region of rat neonatal hippocampus. J Physiol (Lond) 507:219-236.

Ho S, O'Donovan MJ (1992) Optical and pharmacological studies of propriospinal neurons involved in rhythmic motor activity in the embryonic chick spinal cord. Soc Neurosci Abstr 18:1057.

Ho S, O'Donovan MJ (1993) Regionalization and intersegmental coordination of rhythm-generating networks in the spinal cord of the chick embryo. J Neurosci 13:1345-1371.

Katz LC, Shatz CJ (1996) Synaptic activity and the construction of cortical circuits. Science 274:1133-1138.

Lee MT, O’Donovan MJ (1991) Organization of hindlimb muscle afferent projections to lumbosacral motoneurons in the chick embryo. J Neurosci 11:2564-2573.

Lev-Tov A, Pinco M (1992) In vitro studies of prolonged synaptic depression in the neonatal rat spinal cord. J Physiol (Lond) 447:149-169.

Magnuson DSK, Schramm MJ, MacLean JN (1995) Long-duration, frequency-dependent motor responses evoked by ventro-lateral funic- 
ulus stimulation in the neonatal rat spinal cord. Neurosci Lett 192:97-100.

Necker R (1992) Spinal neurons projecting to anterior or posterior cerebellum in the pigeon. Anat Embryol (Berl) 185:325-334.

Nishimaru H, Iizuka M, Ozaki S, Kudo N (1996) Spontaneous motoneuronal activity mediated by glycine and GABA in the spinal cord of rat fetuses in vitro. J Physiol (Lond) 497:131-143.

Obata K, Oide M, Tanaka H (1978) Excitatory and inhibitory actions of glycine on embryonic chick spinal neurons in culture. Brain Res 144:179-184.

O'Donovan MJ (1989) Motor activity in the isolated spinal cord of the chick embryo: synaptic drive and firing pattern of single motoneurons. J Neurosci 9:943-958.

O'Donovan MJ (1999) The origin of spontaneous activity in developing networks of the vertebrate nervous system. Curr Opin Neurobiol 9:94-104.

O'Donovan MJ, Chub N (1997) Population behavior and selforganization in the genesis of spontaneous rhythmic activity by developing spinal networks. Semin Cell Dev Biol 8:21-28.

O'Donovan MJ, Ritter A (1995a) Rhythmic activity patterns of motoneurons and interneurons in the embryonic chick spinal cord. In: Neural control of movement (Ferrell WR, Proske U, eds), pp 195-201. New York: Plenum.

O’Donovan MJ, Ritter A (1995b) Optical recording and lesioning of spinal neurones during rhythmic activity in the chick embryo spinal cord. In: Alpha and gamma motor systems (Taylor A, Gladden MH, Durbaba R, eds), pp 557-563. New York and London: Plenum.

O'Donovan MJ, Sernagor E, Sholomenko G, Ho S, Antal M, Yee W (1992) The development of spinal motor networks in the chick embryo. J Exp Zool 261:261-273.

O'Donovan MJ, Ho S, Yee W (1994) Calcium imaging of rhythmic network activity in the developing spinal cord of the chick embryo. J Neurosci 14:6354-6369.

O’Donovan MJ, Chub N, Wenner P (1998a) Mechanisms of spontaneous activity in developing spinal networks. J Neurobiol 37:131-145.

O'Donovan MJ, Wenner P, Chub N, Tabak J, Rinzel J (1998b) Mechanisms of spontaneous activity in the developing spinal cord and their relevance to locomotion. Ann NY Acad Sci 860:130-142.

Oppenheim RW, Shneiderman A, Shimizu I, Yaginuma H (1988) Onset and development of intersegmental projections in the chick embryo spinal cord. J Comp Neurol 275:159-180.

Perrins R, Roberts A (1995) Cholinergic and electrical motoneuron-to- motoneuron synapses contribute to on-cycle excitation during swimming in Xenopus embryos. J Neurophysiol 73:1005-1012.

Pinco M, Lev-Tov A (1994) Synaptic transmission between ventrolateral funiculus axons and lumbar motoneurons in the isolated spinal cord of the neonatal rat. J Neurophysiol 72:2406-2419.

Ritter A, O’Donovan MJ (1993) Firing patterns and membrane properties of rhythmically active interneurones in the embryonic chick spinal cord. Soc Neurosci Abstr 19:557.

Schwartz TH, Rabinowitz D, Unni V, Kumar VS, Smetters DK, Tsiola A, Yuste R (1998) Networks of coactive neurons in developing layer 1. Neuron 20:541-552.

Sernagor E, O'Donovan MJ (1991) Whole-cell patch clamp recordings from rhythmically active motoneurons in the isolated spinal cord of the chick embryo. Neurosci Lett 128:211-216.

Sernagor E, Chub N, Ritter A, O'Donovan MJ (1995) Pharmacological characterization of the rhythmic synaptic drive onto lumbosacral motoneurons in the chick embryo spinal cord. J Neurosci 15:7452-7464.

Sholomenko G, O’Donovan MJ (1995) Development and characterization of pathways descending to the spinal cord in the embryonic chick. J Neurophysiol 73:1223-1233.

Webster DMS, Steeves JD (1991) Funicular organization of avian brainstem-spinal projections. J Comp Neurol 312:467-476.

Wenner P, O'Donovan MJ (1995) The role of spinal motoneurons and interneurons in the production of rhythmic activity investigated using a new technique for selective inactivation of neuronal populations. Soc Neurosci Abstr 21:688.

Wenner P, O'Donovan MJ (1996) Spontaneous rhythmic activity in spinal cord preparations following surgical ablation of motoneurons. Soc Neurosci Abstr 22:1377.

Wenner P, Matise M, Joyner A, O’Donovan MJ (1998) Physiological and molecular characterization of interneurons in the developing spinal cord. Ann NY Acad Sci 860:425-427.

Wong RO, Chernjavsky A, Smith SJ, Shatz CJ (1995) Early functional neural networks in the developing retina. Nature 374:716-718.

Wu W, Ziskind-Conhaim L, Sweet MA (1992) Early development of glycine- and GABA-mediated synapses in rat spinal cord. J Neurosci 12:3935-3945.

Yaginuma H, Shiga T, Homma S, Rieko I, Oppenheim R (1990) Identification of early developing axon projections from spinal interneurons in the chick embryo with a neuron specific b-tubulin antibody: evidence for a new "pioneer" pathway in the spinal cord. Development 108:705-716. 